OPEN ACCESS

Edited by:

Abel Santos,

University of Adelaide, Australia

Reviewed by:

Tran Thanh Tung,

University of Adelaide, Australia

Soong Ju Oh

Korea University, Japan

*Correspondence:

Ho Won Jang

hwjang@snu.ac.kr

Specialty section: This article was submitted to

Nanoscience,

a section of the journal

Frontiers in Chemistry

Received: 11 August 2019

Accepted: 09 October 2019

Published: 15 November 2019

Citation:

Lee CW, Suh JM and Jang HW (2019)

Chemical Sensors Based on

Two-Dimensional (2D) Materials for

Selective Detection of Ions and

Molecules in Liquid.

Front. Chem. 7:708

doi: 10.3389/fchem.2019.00708

\section{Chemical Sensors Based on Two-Dimensional (2D) Materials for Selective Detection of lons and Molecules in Liquid}

\author{
Chung Won Lee, Jun Min Suh and Ho Won Jang* \\ Department of Materials Science and Engineering, Research Institute of Advanced Materials, Seoul National University, \\ Seoul, South Korea
}

Up until now, two-dimensional (2D) materials have been researched vigorously for application to sensing ions and molecules in liquid due to their unique structural, chemical, and electronic properties. Features of 2D materials such as high surface area-to-volume ratios and various reaction sites are ideal characteristics for fabricating state-of-the-art high-performed chemical sensors. This review particularly focuses on the detection of $\mathrm{pH}$, metal ions, and biomolecules in liquid media. The final goal of the ion/molecule sensors is a development of the electronic tongue or taste sensors that can be used in medical, food, biotechnology, and health applications. Herein, we introduce recent advances in the field of ion/molecule sensors in liquid media based on 2D materials, especially concentrating in graphene and $\mathrm{MoS}_{2}$, and will emphasize the opportunities and challenges of these unique sensing materials and devices.

Keywords: liquid, chemical sensor, biosensor, graphene, transition metal dichalcogenides, 2D materials

\section{INTRODUCTION}

A recent development in sensor technology and increasing demands in high-quality life have led to the era of the Internet of Things (IoT). The interconnected sensor devices and numerous data acquisition from various sources including the human body, indoor air, foods, infrastructures, and so on can provide real-time information of harmful substances or human body status with adequate actions to take upon those signals (Nowogrodzki, 2018). Among various fields of sensor technologies, the development of smart sensors mimicking human organs has attracted a tremendous amount of attention since electronically replicated human organs can examine various target substances with extremely lower detection limit and higher sensitivity, leading to early detection of the harmful agents. The human five senses include sight, hearing, smell, taste, and touch, and they respectively have a different approach to be electronically replicated in various research fields. The sight and hearing sensors have already been developed to a high level enough to be commercialized such as eye-tracking technologies and speech recognition in mobile phones and automobiles (Itti, 2015). The remaining smell, taste, and touch senses also have been intensively studied to develop sensors mimicking the human nose, tongue, and skin but still require further researches to be in real use as sight and hearing are.

Among the remaining three human senses, there has been significant progress in developing an electronic tongue (e-tongue) mimicking the human taste sense. Unlike electronic nose or skin aiming in various gaseous substances and external stimuli, respectively, e-tongue targets distinctive 
ions, or molecules (glucose, hydrogen ion, quinine, sodium ion, and monosodium glutamate) representing each of the five tastes (sweet, sour, bitter, salty, and umami). Although some companies have already commercialized equipment analyzing the above five taste substances, they have a relatively large scale to be adopted into the IoT platform, where small size, low power consumption, low price, and compatibility to existing circuits are essential requirements.

The two-dimensional (2D) materials are emerging candidates to be used in sensing devices and, further, can be applied to the IoT platform due to their promising physical and chemical properties. Their high surface area-to-volume ratio leads to high sensitivity to target substances. 2D materialbased sensors can also be fabricated in a miniaturized size due to their flexibility, great mechanical strength, and optical transparency (Novoselov et al., 2005; Castellanos-Gomez et al., 2010; Gorbachev et al., 2011; Wang et al., 2012; Butler et al., 2013). For practical use of $2 \mathrm{D}$ material-based sensors, many requirements should be considered, but the selectivity issue should be the primary consideration. The approaches in the aspect of characteristics of sensing materials are the most effective way to improve ions or molecule selectivity since the selectivity highly depends on the nature of $2 \mathrm{D}$ materials and ion/molecule. 2D materials interact with target molecules/ions by two distinctive mechanisms: physisorption and chemisorption. Physisorption happens when the molecules/ions interact with the surface of 2D materials without any covalent bonding, and chemisorption refers to the covalent interactions between molecules/ions and the surface of $2 \mathrm{D}$ materials. When noncovalent interactions are preferred, it results in quick response and fast recovery. However, biomolecules such as DNA requires immobilizing processes on the surface, which makes covalent linking more preferable. The physisorption of molecules/ions onto 2D materials surface depends on the properties of both analyte and surface. For example, graphene has a honeycomb structure with a linkage of $\mathrm{sp}^{2}$ hybridized carbon atoms by a longrange $\pi$-conjugation. As a result, the non-covalent interaction between the surface of graphene and ions/molecules involving $\pi$ bonds is important since the electronic characteristics of $\pi$ systems can modify the structure or properties (Georgakilas et al., 2012). On the other hand, a representative transition metal dichalcogenide (TMD), $\mathrm{MoS}_{2}$, exhibits physisorption onto its basal planes due to the interactions between the ion/molecule and $\mathrm{MoS}_{2}$ via van der Waals force (Moses et al., 2009). Chemisorption can be induced by forming defects on the surface of the $2 \mathrm{D}$ materials. For example, graphene has point defects such as the absence of some $\mathrm{sp}^{2}$ carbon atoms or carbon atoms having $\mathrm{sp}^{3}$ hybridization. These defects induce different electronic structures from the original $\mathrm{sp}^{2}$ carbons resulting in an alteration of chemical reactions. These point defects increase the chemical reactivity of graphene. Furthermore, defects can be exploited to form nano-sized pores, permselective membranes (i.e., ion exchanger), or ultrasensitive sensors that can detect a sequence of DNA. Chemical functionalization of 2D materials can also enhance selectivity toward target substances.

Herein, we summarized various studies and efforts on developing ion/molecule sensors based on $2 \mathrm{D}$ materials, especially focusing on graphene and $\mathrm{MoS}_{2}$; our scope is illustrated in Figure 1. Due to promising characteristics of $2 \mathrm{D}$ materials as above, their application to sensors has been conducted explosively in various approaches targeting numerous substances. Although there have been good reviews on $2 \mathrm{D}$ material-based sensors targeting gaseous molecules, only a few summarized those targeting ions and molecules existing in liquid media, which require much more complicated sensing mechanism and sensor structures. In this review, various 2D material-based sensors targeting ions and molecules including hydrogen ion, glucose, metal ions, organic molecules, and biomolecules are summarized with their sensor performances in the platforms of field effect transistors to help understand the current progress of the state-of-the-art ion/molecule sensors based on $2 \mathrm{D}$ materials.

\section{HYDROGEN ION SENSING}

The most fundamental ion/molecule sensor is the hydrogen ion $\left(\mathrm{H}^{+}\right)$sensor (i.e., $\mathrm{pH}$ sensor). Hydrogen ion sensing is a vital measurement in many liquid chemical procedures such as industrial, manufacturing, pharmaceutical, and food production. The acidity of a substance may act as an indicator in many cases; food spoilage indication, monitoring clean/wastewater, chemical laboratory analysis, etc. $\mathrm{pH}$ meters commonly used in scientific laboratories are also called potentiometric $\mathrm{pH}$ meters. The basic fundamental of $\mathrm{pH}$ meters is measuring the electrical potential difference and monitoring the hydrogen ion concentration of a typical solution. This method is very precise in sensing the concentration of hydrogen ions, but there is a critical disadvantage that the device is not portable due to its size and the necessity of external power source. Explained above, IoT devices must be miniaturized and portable for everyday use. $2 \mathrm{D}$ materials can be used as $\mathrm{pH}$ sensors because of their high sensitivity from their chemical and physical properties. Additionally, hydrogen ion sensors based on 2D materials can be minimized in size, making them emerging candidates of hydrogen ion sensors.

\section{Graphene}

Graphene can electrically detect chemical species due to its single-layer thickness and high carrier mobility (Ohno et al., 2009; Cheng et al., 2010). Since graphene has a unique semimetallic characteristic with a zero bandgap (Berger et al., 2006), graphene field-effect transistors (GFETs) reveal bipolar features in terms of conduction properties. GFETs exhibit distinct transfer characteristics in source-drain current vs. gate voltage $\left(I-V_{\mathrm{g}}\right)$, which has a conductance minimum point called Dirac point ( $V_{\text {Dir }}$ ) or charge neutrality point $\left(V_{\mathrm{CNP}}\right)$. In the case of $V_{\mathrm{g}}$ having a lower value than $V_{\mathrm{CNP}}$, the majority of charge carriers are holes and increasing $V_{\mathrm{g}}$ leads to the decline of channel current and, reversely, the majority charge carriers become electrons, which increases in quantity with increasing $V_{\mathrm{g}}$. Consequently, GFETs have the advantage of noticing the shift of charge neutrality point by adsorption or desorption of electrons or charged ions. Sensitivity can be estimated by evaluating the degree of horizontal shift of the charge neutrality point. In other words, as chemical alteration such as ion concentration or $\mathrm{pH}$ increase 


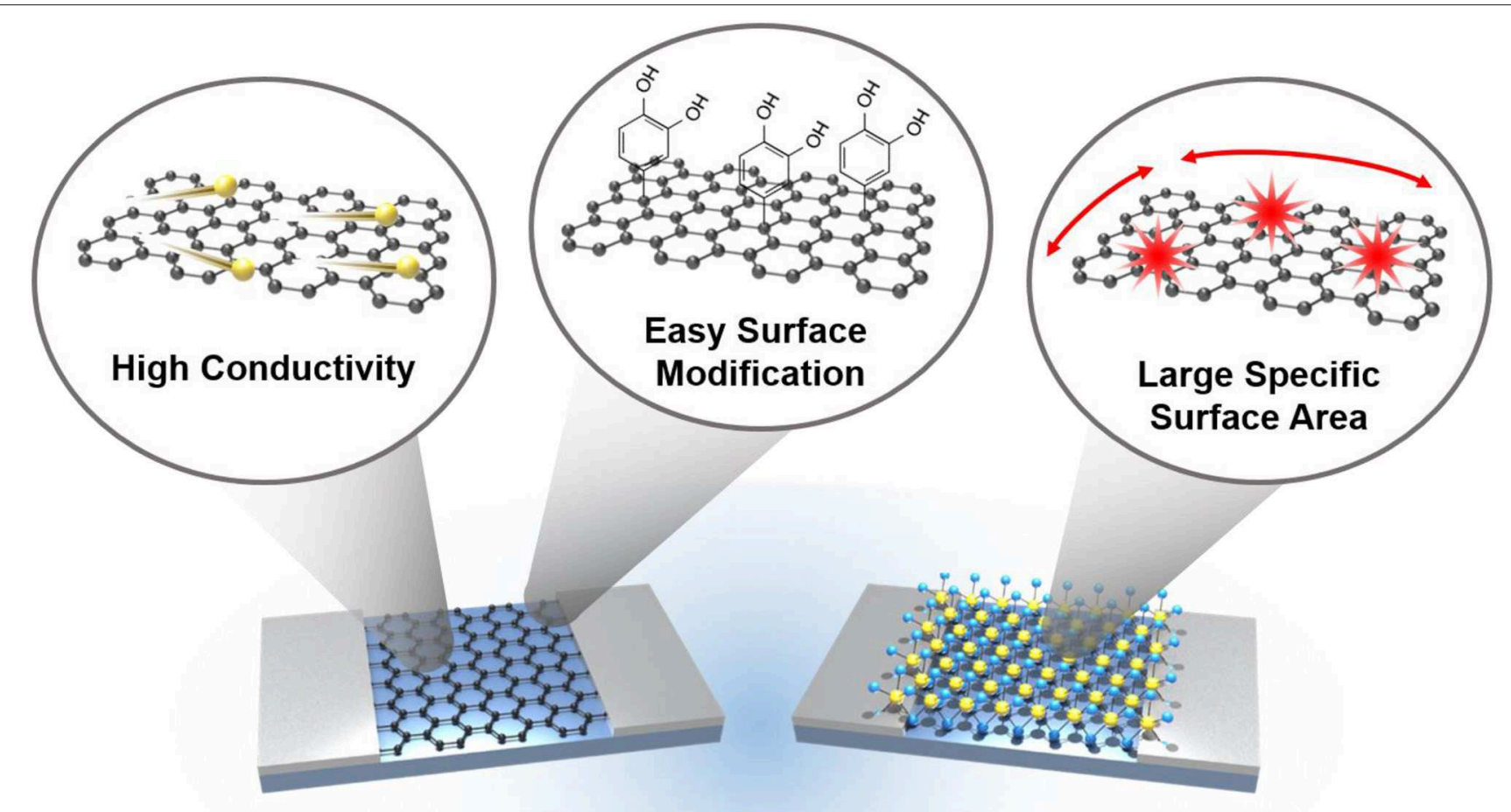

\section{Chemical Sensors based on 2D Materials}

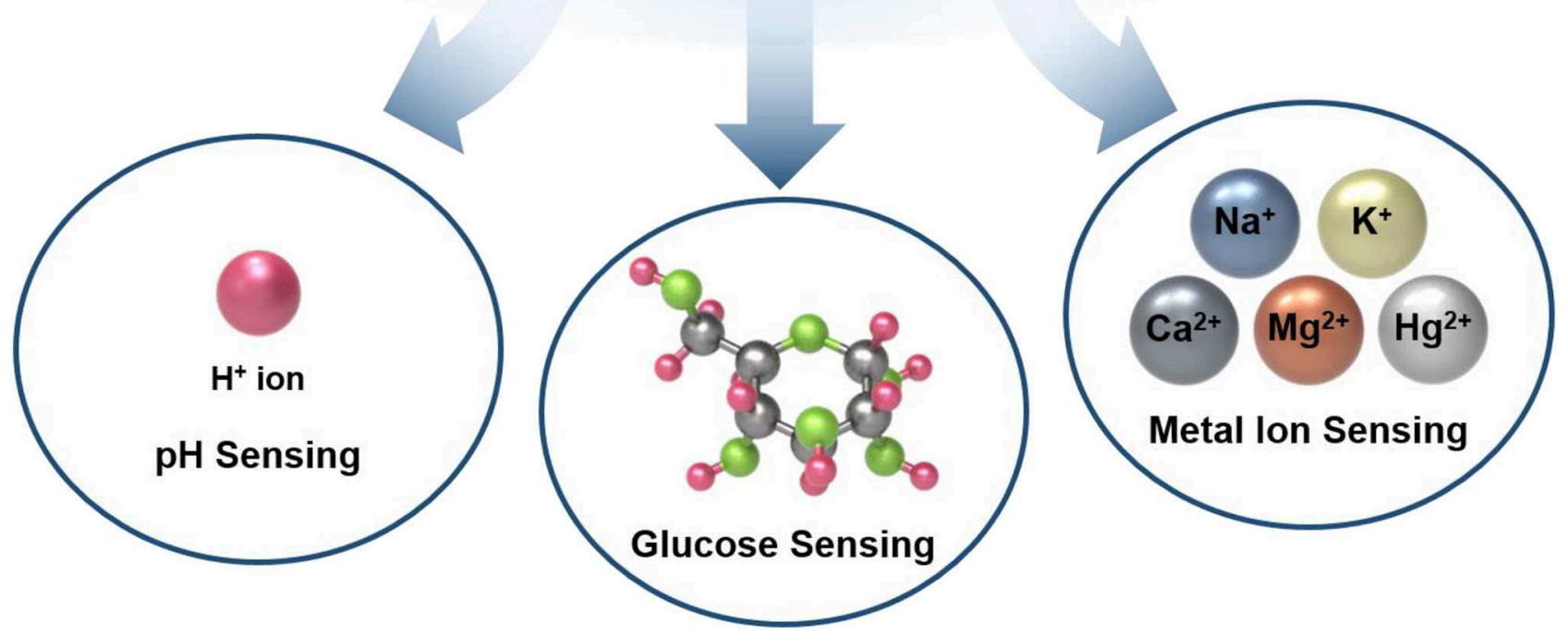

FIGURE 1 | Schematic illustration of chemical sensors based on 2D materials with advantages of 2D materials and their application into ion/molecule sensing.

or decrease results in the surface of GFET, the $V_{\mathrm{CNP}}$ will change (Kwon et al., 2015).

Ohno et al. successfully demonstrated the transport behaviors of GFETs in $\mathrm{pH}$ solutions and protein molecules (Ohno et al., 2009). The prepared graphene was a single-layered crystal prepared by mechanically exfoliating natural graphite. Figure $\mathbf{2 A}$ indicates the optical microscope image of exfoliated graphene contacted with gold electrodes and Figure 2B depicts the overall setup of the experiment. The input electrolytes ( $\mathrm{pH}$ solutions) into the well were made with mixing various types of buffer solutions for each $\mathrm{pH}$ value. The relationship between transfer characteristics and conductance of GFET on $\mathrm{pH}$ was revealed. By the shift of charge neutrality point toward the positive direction as the increment of $\mathrm{pH}$, it could be assumed that the negative charge needed to reach the neutral point (minimum value) increases as $\mathrm{pH}$ rises since holes are the main charge carrier. Cheng et al. reduced the noise level and improved the signalto-noise ratio by removing the oxide by suspending graphene 
in aqueous solutions for in situ etching (Cheng et al., 2010). After mechanical exfoliation of graphite, single-layer graphene was transferred to the silicon substrate. An etching process was progressed, preventing graphene from air exposure because any drying steps will generate stress and eventually deform the graphene. The deformation of the graphene will alter the electrical properties of it and will consequently cause graphene to malfunction. Buffed hydrofluoric acid (HF) was used for etching and the conductance of the device drastically declined after injection. After the drastic decrease of conductivity, it was stabilized in 50 to $100 \mathrm{~s}$, designating the termination of the etching process. As shown in Figure $\mathbf{2 C}$, transfer curves were depicted in the span of $\mathrm{pH} 6$ to 9. These $\mathrm{pH}$ solutions are prepared with a combination of potassium chloride and phosphate solution. Charge neutrality points are shifted to the right as $\mathrm{pH}$ increases in Figure 2C. Conductivity value increases as $\mathrm{pH}$ increases in negative $V_{\mathrm{g}}$. But tendency reverses if the $V_{\mathrm{g}}$ has a positive value as shown in Figure 2D. This result can be confirmed in the graph of Figure 2C. In $V_{\mathrm{g}}=-0.05 \mathrm{~V}$, conductivity is higher in higher $\mathrm{pH}$, and in $V_{\mathrm{g}}=0.05 \mathrm{~V}$, conductivity is lower in higher $\mathrm{pH}$. This is due to the ambipolar characteristics of graphene; mentioned above, in the case of $V_{\mathrm{g}}<V_{\mathrm{CNP}}$, the main charge carrier are holes and higher $\mathrm{pH}$ will result in higher conductivity because $\mathrm{OH}^{-}$ions induce holes to the surface of graphene. Reversely, in the case of $V_{\mathrm{g}}$ $>V_{\mathrm{CNP}}$, the main charge carriers are electrons and lower $\mathrm{pH}$ will result in higher conductivity because hydrogen ions induce electrons to the surface of graphene. Reversibility was confirmed by repeatedly injecting $\mathrm{pH}$ solutions in sequence. Zhu et al. designed a GFET without an external gate source (elimination of reference rod) by embedding a solid gate, suggesting the possibility of a practical analytic device using graphene (Zhu et al., 2015). A typical thin layer composed of $\mathrm{HfO}_{2}$ with a high dielectric constant $(\kappa)$ is used as a gate dielectric layer. The $\mathrm{HfO}_{2}$ layer has higher specific capacitance compared to conventional $\mathrm{SiO}_{2}$ solid-gated sensors, leading to higher transconductance and operation at lower $V_{\mathrm{g}}$. Also, the $\mathrm{HfO}_{2}$ layer prevents the gate electrode to be exposed from the liquid, eliminating any errors (e.g., bulk motion of sample solution). This device does not require any separate components such as external reference rods, which simplifies the fabrication process. Using a low $V_{\mathrm{g}}(1.5 \mathrm{~V})$, the device can measure $\mathrm{pH}$ in a span of 5.3 to 9.3 with a sensitivity of $\sim 57 \mathrm{mV} / \mathrm{pH}$.

Graphene oxide (GO) and reduced graphene oxide (RGO) are also candidates for $\mathrm{pH}$ sensing. $\mathrm{GO}$ is a $2 \mathrm{D}$ material that is originated from natural graphene by chemical conversion (Park and Ruoff, 2009). GO has various advantages such as being stable into single-layered sheets in water and being functionalized with ease. Also, GO is differentiated to graphene by its semiconducting features from the oxidation of graphene, leading to bandgap increase (Cai et al., 2008). There are various methods to synthesize GO such as the Staudenmaier (1898) and Hummers method (Hummers Jr and Offeman, 1958). These methods all include the oxidation process of graphite. To oxidize graphite, Staudenmaier mixed potassium chlorate $\left(\mathrm{KClO}_{3}\right)$ with nitric acid $\left(\mathrm{HNO}_{3}\right)$. On the other hand, Hummers treated graphite with a mixture of potassium permanganate $\left(\mathrm{KMnO}_{4}\right)$ and sulfuric acid $\left(\mathrm{H}_{2} \mathrm{SO}_{4}\right)$ (Zhu et al., 2010). Due to the polar oxygen functional groups of $\mathrm{GO}$, exfoliation in many solvents is possible and dispersion by simple stirring or sonication is available with ease (Zhu et al., 2010). RGO has similar properties with graphene since the reduction process on GO involves removing oxygen functional groups. In other words, RGO possesses both advantages of pure graphene and GO: high conductivity and existence of chemically active defect sites (Robinson et al., 2008). There are a variety of methods to reduce $\mathrm{GO}$ to RGO; exposing GO to reducing chemicals such as hydrazine or to alkalis, hydrogen plasma treatment, thermal annealing, and photocatalytic reduction (Stankovich et al., 2006; Gómez-Navarro et al., 2007; Jung et al., 2008; Wang et al., 2008; Williams et al., 2008). GO and RGO are typically p-type materials, so when these materials are exposed to oxidizing gases (e.g., $\mathrm{NO}, \mathrm{NO}_{2}$ ), the resistance of both materials declines (Choi et al., 2015).

The most distinct feature of GO compared to pristine graphene and RGO is that, GO is electrically insulating (Fan et al., 2008) and a bandgap exists (Boukhvalov and Katsnelson, 2008; Eda et al., 2010). Due to these characteristics, rather gathering data with transfer curves or current vs. time curves, GObased $\mathrm{pH}$ sensors are focused on luminescence features taking advantage of the presence of bandgap. Chen et al. revealed the reversibility of vis-NIR fluorescence under different conditions of acidic and salt $\left(\mathrm{Na}^{+}\right)$conditions or $\mathrm{pH}$ (Figures 3A,B; Chen and Yan, 2011). The protonation and deprotonation of the carboxylate functional group from GO lead to activation and deactivation of luminescence. When the state of the carboxyl functional group is $-\mathrm{COOH}$, it becomes luminescent and if the state becomes $-\mathrm{COO}^{-}$, it alters to non-luminescent. Adding $\mathrm{OH}^{-}$to $-\mathrm{COOH}$ will induce it to become $-\mathrm{COO}^{-}$, and on the contrary, applying $\mathrm{H}^{+}$to $-\mathrm{COO}^{-}$will change it to $-\mathrm{COOH}$. To summarize, adding salt or $-\mathrm{OH}^{-}$leads luminescence intensity to decrease. On the contrary, removing salt or applying $\mathrm{H}^{+}$ leads to luminescence intensity to increase. As depicted in Figures $\mathbf{3 A}, \mathbf{B}$, both reactions are reversible and eventually find their initial states. Bai et al. formed a composite hydrogel with GO and poly(vinyl alcohol) (PVA) for selective drug release at a particular pH (Bai et al., 2010). Various content ratio of GO/PVA mixtures was produced to find the ideal proportion. Theoretically, one PVA chain can be linked with two or more GO sheets, which can form cross-linking sites. If PVA content exceeds the ratio of 1:2 (=PVA: GO), the majority of PVA chains become adsorbed on both faces of a single-layer GO, which leads to weakening the cross-linking effect of PVA chains. The ideal ratio was 1:10 (=PVA: GO) for releasing drugs at physiological $\mathrm{pH}$. The drug leakage ability of the GO/PVA composite was evaluated by loading vitamin $\mathrm{B}_{12}$ to the hydrogel and testing in various $\mathrm{pH}$ conditions. Rather than acidic conditions, releasing in neutral conditions have higher efficiency. Due to the $\mathrm{pH}$ sensitive properties of GO, drugs can be released to preferred physiological $\mathrm{pH}$.

Possessing both merits of pristine graphene and GO, RGO is also a candidate for $\mathrm{pH}$ sensors. The surface and edges on the RGO nanosheets contain oxygen functional groups that will 

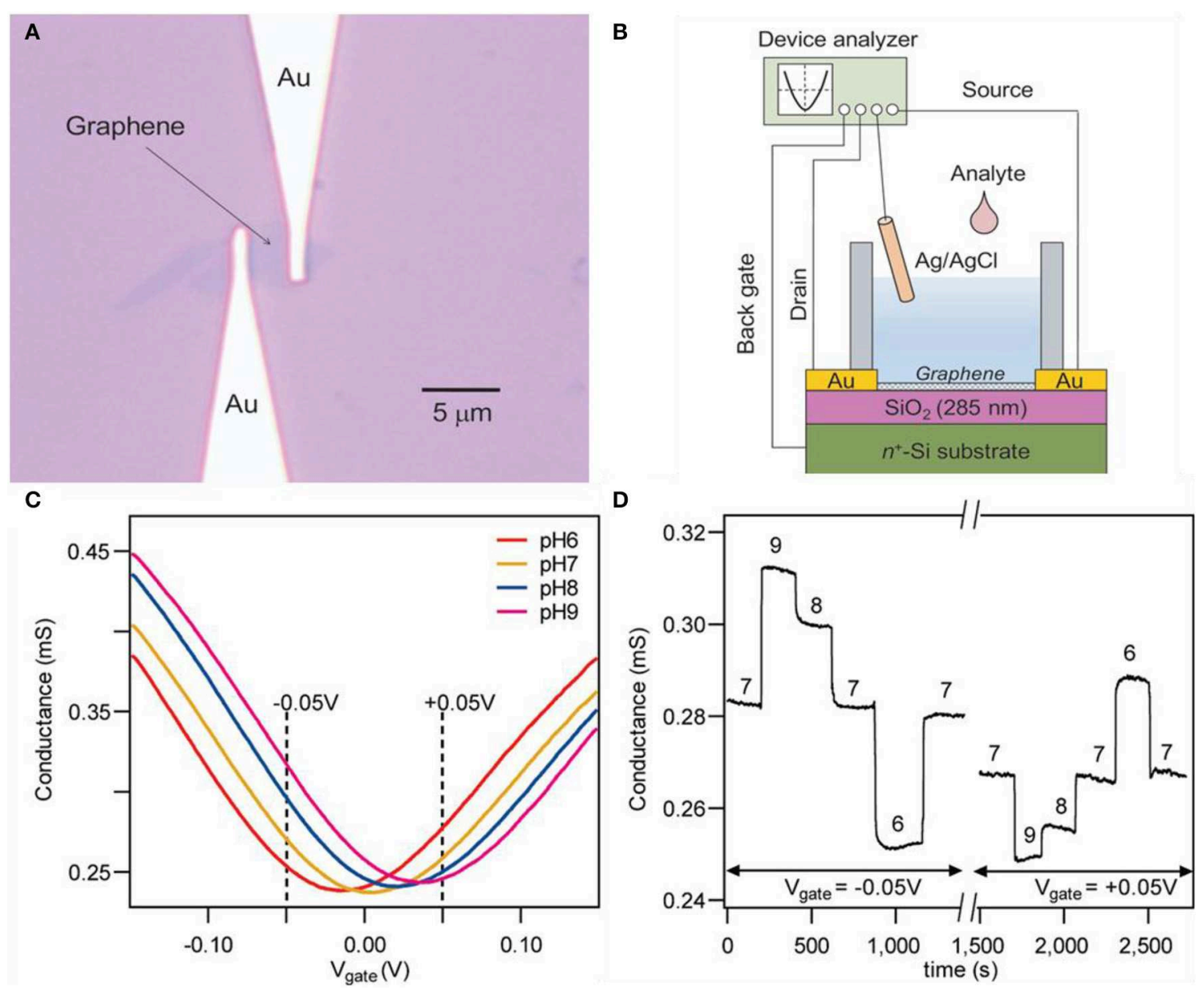

FIGURE 2 | Pristine graphene pH sensors. (A) Optical microscope image of a graphene field effect transistor. (B) Experimental setup scheme illustration of a graphene field effect transistor. Figures (A,B) were reproduced from Ohno et al. (2009) with permission from the American Chemical Society. (C) Typical transfer curves and (D) time-current curves of a graphene field effect transistor. Figures (C,D) were reproduced from Cheng et al. (2010) with permission from the American Chemical Society.

enhance the sensing response of $\mathrm{pH}$ (Sohn et al., 2013). Sohn et al. reduced manufactured GO nanosheets by the Hummers method. After the exfoliation process of GO by sonication, it was spin-coated to the $\mathrm{SiO}_{2}$ substrate and a channel area was formed. After the drying process, the GO was reduced with hydrazine monohydrate to produce a conductive RGO channel. After annealing at $200^{\circ} \mathrm{C}$ for $2 \mathrm{~h}$ in Ar atmosphere, for additional functional groups, the RGO channel was immersed in dimethylformamide (DMF) containing 1-pyrenebuthanoic acid succinimidyl ester at room temperature (RT) for $2 \mathrm{~h}$. Buffer solutions were used by mixing $0.1 \mathrm{M}$ phosphate buffer and $0.1 \mathrm{M}$ $\mathrm{NaCl}$ solution. Figure 3C illustrates the overall setup of the RGO channel device. The sensing curves of the RGO channel are depicted in Figure 3D, having lower sensitivity toward lower $\mathrm{pH}$. Pristine graphene also has an identical tendency of RGO in terms of sensing $\mathrm{pH}$. Their sensing mechanism is equal in the point of view of $\mathrm{H}^{+}$binding to the surface, eventually inducing the surface charge density to change on the gate. Precisely, the processes are different. In the case of pristine graphene, when exposed to acid electrolytes, hydronium ions $\left(\mathrm{H}_{3} \mathrm{O}^{+}\right)$attach to the inner
Helmholtz plane (interface formed when an electrolyte and electronic conductor meets), inducing the graphene to become electron doped. On the other hand, when exposed to alkali electrolyte, hydroxyl $\left(\mathrm{OH}^{-}\right)$attaches to the inner Helmholtz plane and the graphene becomes hole doped (Lei et al., 2011). Both $\mathrm{H}_{3} \mathrm{O}^{+}$and $\mathrm{OH}^{-}$ions are non-faradic (capacitive), so these charges are impermeable across the graphene/electrolyte surface (Ang et al., 2008). As the $\mathrm{H}_{3} \mathrm{O}^{+}$ions or $\mathrm{OH}^{-}$ions are distributed to the Helmholtz inner plane, $\mathrm{OH}^{-}$ions are more orderly arranged in the inner plane than $\mathrm{H}_{3} \mathrm{O}^{+}$ions. Therefore, the conductivity value has a proportional relationship with $\mathrm{pH}$ (Ang et al., 2008). RGO also has functional groups such as -OH, $\mathrm{COOH}$ on the surface similar to $\mathrm{GO}$ and high conductivity, which is the keystone of the sensing mechanism. These groups have interactions with $\mathrm{H}^{+}$ions in the electrolyte. In lower $\mathrm{pH}$ buffer solutions, more $\mathrm{H}^{+}$combines with $\mathrm{O}^{-}$and $\mathrm{COO}^{-}$, consequently leading the positive charge to incline on the surface of RGO. This induces the increment of electrons in the channel, which leads the $V_{\mathrm{CNP}}$ to move in the negative direction (Sohn et al., 2013). 


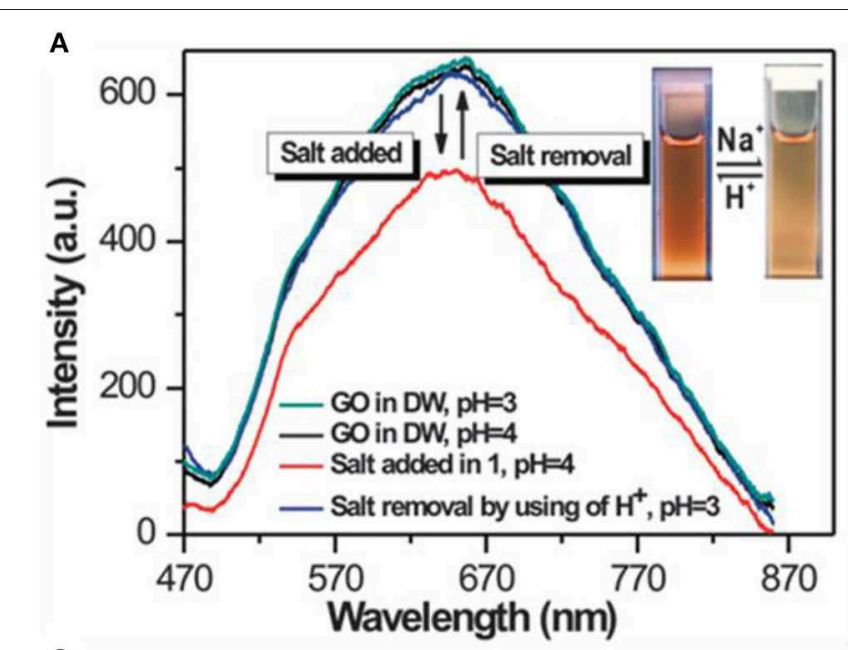

C

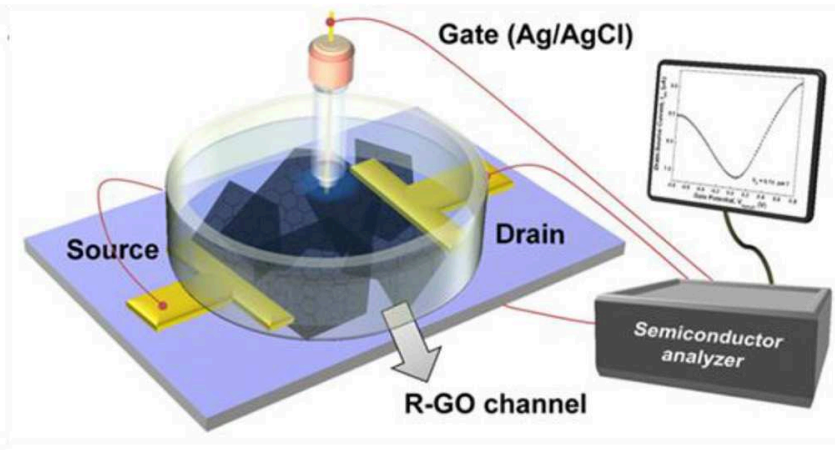

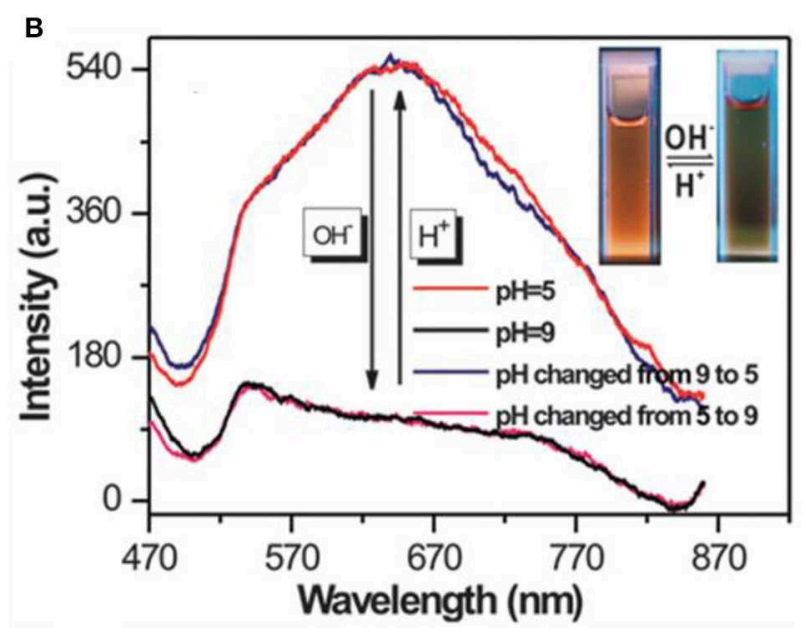

D

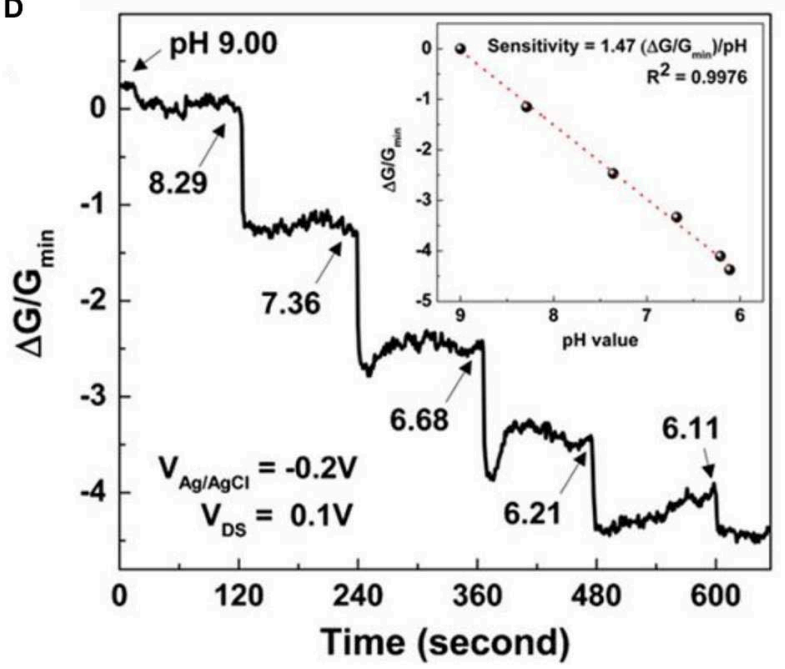

FIGURE 3 | Graphene oxide (GO)- and reduced graphene oxide (RGO)-based pH sensors. (A) Reversible vis-NIR fluorescence change of acidic vs. salt states. (B) Reversible vis-NIR fluorescence change of $\mathrm{pH}$. Figures (A,B) were reproduced from Chen and Yan (2011) with permission from the Royal Society of Chemistry. (C) Schematic diagram of an RGO field effect transistor. (D) Typical sensing curves along pH alter RGO. Figures (C,D) were reproduced from Sohn et al. (2013) with permission from Elsevier.

\section{TMDs and Other 2D Materials}

The most representative TMD is molybdenum disulfide $\left(\mathrm{MoS}_{2}\right)$, composed of Mo (transition metal) and S (chalcogenide atom), which are covalently bonded. These $2 \mathrm{D}$ stacked layers are arranged in a row and they are held together by weak van der Waals force. This weak force makes exfoliation rather easier, and single-layered film extraction is possible (Sarkar et al., 2014). The mechanism of TMD-based devices detecting $\mathrm{pH}$ alteration is similar to GFET. Sarkar et al. measured the current change as $\mathrm{pH}$ alters and proposed $\mathrm{MoS}_{2}$ as a candidate for a typical $\mathrm{H}^{+}$ion sensor (Sarkar et al., 2014). As shown in Figure 4A, the main sensing mechanism is the protonation and deprotonation of the $\mathrm{OH}$ groups on the surface of the gate dielectric by the $\mathrm{pH}$ of the electrolyte. In the case of low $\mathrm{pH}$, the surface will be protonated $\left(\mathrm{OH}+\mathrm{H}^{+}=\mathrm{OH}_{2}^{+}\right)$, generating positive charges while deprotonation occurs on the surface $\left(\mathrm{OH}-\mathrm{H}^{+}=\mathrm{O}^{-}\right)$in high $\mathrm{pH}$ resulting in negative charges. In Figure 4B, electrolyte gate voltage vs. drain current curves is depicted for various $\mathrm{pH}$ values. At a fixed applied bias, the decline of $\mathrm{pH}$ value will lead to the incline of current (i.e., dielectric surface having a higher positive charge inducing to lower the threshold voltage of FET) and vice versa. This threshold voltage shift can be explained by surface charge alter due to $\mathrm{pH}$ change. Liao et al. used layered rhenium disulfide $\left(\operatorname{ReS}_{2}\right)$ for low-frequency noise field effect transistors for $\mathrm{pH}$ sensing (Liao et al., 2018). The $\mathrm{ReS}_{2}$ flakes were obtained by mechanically exfoliating commercial bulk $\mathrm{ReS}_{2}$ crystals. Illustrated in Figure 4C, the diagram reveals the overall structure of $\mathrm{ReS}_{2}$. An $\mathrm{Ag} / \mathrm{AgCl}$ electrode was used for the liquid gate and a thin $\mathrm{HfO}_{2}$ gate dielectric was used to hold phosphate buffered saline (PBS) solution, respectively. The thickness of $\mathrm{HfO}_{2}$ was $20 \mathrm{~nm}$, which was deposited by atomic layer deposition (ALD) on highly doped Si. Figure 4D indicates the transfer curves of the $\mathrm{pH}$ sensor, which shifts in a positive direction as the $\mathrm{pH}$ value increases. Higher $\mathrm{pH}$ value in the 
solution induces a more negative charge on the surface of the $\mathrm{HfO}_{2}$ gate. Figure 4E shows response curves ( $I_{\mathrm{DS}}$ vs. time) on various $\mathrm{pH}$ values. The source-drain and the gate voltage are fixed to $V_{\mathrm{DS}}=0.1 \mathrm{~V}$ and $V_{\mathrm{LG}}=-0.5 \mathrm{~V}$, and current changes drastically and stabilizes quickly in each $\mathrm{pH}$ value. Shadman et al. used $\mathrm{MoS}_{2}$ and $\mathrm{WSe}_{2}$ as a double gate field effect transistor for $\mathrm{pH}$ sensing (Shadman et al., 2016). The sensitivity is influenced by the thickness of back and top oxide. The thicker the back oxide and the thinner the top oxide, the higher the sensitivity of the device. Nasir et al. investigated the alteration of inherent oxidative peaks in various $\mathrm{pH}$ values of different TMDs (Zafir Mohamad Nasir et al., 2015). Using potential-pH diagrams (Pourbaix diagram), this group found $\mathrm{MoS}_{2}$ and $\mathrm{MoSe}_{2}$ becoming oxidized from +4 to +6 states at $\mathrm{pH}$ range $\sim 8$. Also, until $\mathrm{pH} 11$, the peak position remains almost stable, but the shift of the peak is considerable. The peak of $\mathrm{WS}_{2}$ was almost similar to the $\mathrm{pH}$ range of 2-12. The tungsten-based dichalcogenides possessed better stability than molybdenum-based dichalcogenides.

\section{GLUCOSE SENSING}

Glucose is a vital component in sitology, physiology, and biotechnology. Glucose is also the most basic and important energy source of human daily life. If glucose concentration decreases in the human body, people may go under shock. On the other hand, an excessive amount of glucose in the blood results in diabetes, which is an enormous problem these days. In both cases, glucose monitoring is crucial, which many researchers are focusing on fast, reliable glucose sensors for healthcare monitoring. In the 1960s, Clark and Updike first reported the enzyme electrode (Clark and Lyons, 1962) and biosensor (Updike and Hicks, 1967), respectively. After these studies, glucose sensors based on glucose oxidase (GOx) have been researched.

\section{Graphene}

Graphene-based materials are promising candidates for glucose sensing, but rather than using pristine graphene, decorating metal nanoparticles or forming a composite form using chitosan is much more favorable for selectivity. Using functionalized GO or RGO is also an alternative. Wang et al. used nitrogendoped graphene for selective detection toward glucose by treating graphene with nitrogen plasma (Wang et al., 2010). The nitrogen percentage was controlled by plasma exposure time, consisting of $0.11-1.35 \%$. N-doped graphene has the advantage of having high electrocatalytic activity for $\mathrm{H}_{2} \mathrm{O}_{2}$ reduction and fast, direct electron transfer to GOx. GOx can catalyze the oxidation process of glucose to gluconic acid and $\mathrm{H}_{2} \mathrm{O}_{2}$ with oxygen. In other words, glucose sensing is equivalent to the detection of $\mathrm{H}_{2} \mathrm{O}_{2}$ during the enzymatic catalytic activity. As shown in Figure 5A, three different electrodes were compared by the performance of the catalytic effect. The measurements on each type were conducted at $-0.15 \mathrm{~V}$ in $0.1 \mathrm{M}$ physiological buffer solution with a consecutive addition of $0.1 \mathrm{M}$ glucose. Also, in Figure 5B, selectivity of sensors was investigated by adding uric acid ( $5 \mathrm{mM}$ ) and ascorbic acid $(5 \mathrm{mM})$. After confirming no response to uric and ascorbic acids, glucose was added from high concentration to low, obtaining linear responses for each concentration. Kwak et al. used chemical vapor deposition (CVD)-grown graphene for glucose sensing (Kwak et al., 2012). The interesting point is that for a flexible device, this group choose polyethylene terephthalate (PET) as a substrate. As illustrated in Figure 5C, conductive silver paste was covered on both ends of the graphene thin film. A well-type polydimethylsiloxane (PDMS) was attached on top of the graphene to confine the solutions from leaking out. Then, graphene was then surface modified with GOx for catalytic effects when sensing glucose. The gate voltage is applied from the top gate electrode, which induces the accumulation of ions at the boundary between graphene and solution. Graphene also has ambipolar features, explained previously on the $\mathrm{pH}$ sensor section, that can be operated both in $\mathrm{n}$ - and p-type regions. If the charge neutrality point $\left(V_{\mathrm{CNP}}\right)$ is higher than the gate voltage $\left(V_{\mathrm{g}}\right)$, graphene is in the circumstance of $\mathrm{p}$ type, and in contrast to this, graphene behaves as an n-type material. Figure 5D indicates that $V_{\mathrm{CNP}}$ is in between $0.1 \mathrm{~V}$ and $1.0 \mathrm{~V}$. In other words, in the case of $V_{\mathrm{g}}=0.1 \mathrm{~V}$, it is $\mathrm{p}$ type, and at $V_{\mathrm{g}}=1.0 \mathrm{~V}$, it is n-type. In both cases, $V_{\mathrm{DS}}=$ $-0.2 \mathrm{~V}$ and an identical amount of glucose was injected with higher concentration each step. Kang et al., Shan et al., and Wu et al. all manufactured the sensor by fabricating a nanocomposite with GOx (enzyme)-graphene-chitosan. Wu et al. additionally added platinum nanoparticles for higher sensitivity while Shan et al. added Au nanoparticles. Kang et al. dispersed graphene in chitosan solution using ultrasonication. Then, GOx was coated on the graphene/chitosan film (Kang et al., 2009). Wu et al. added Pt nanoparticles to enhance the response of glucose compared to the un-decorated sample (Wu et al., 2009). Shan et al. also had a property increment of glucose sensing using the decoration of $\mathrm{Au}$ nanoparticles (Shan et al., 2010).

Using graphene as well as using GO and RGO are alternatives for glucose sensing. In particular, carboxyl-modified GO possesses the ability to intrinsically catalyze the peroxidase reaction (Song et al., 2010). Song et al. noticed the peroxidase process by checking $\mathrm{GO}-\mathrm{COOH}$ turning into blue when catalytic activity happens. The optimal $\mathrm{pH}$ was 4.0 , the temperature was $35^{\circ} \mathrm{C}$, and $150 \mathrm{mM}$ of $\mathrm{H}_{2} \mathrm{O}_{2}$ concentration. Also, this sensor has selectivity to glucose; fructose, lactose, and maltose have no response. Wang et al. performed a typical RGO glucose sensor by electrochemically reducing GO (Wang et al., 2009). GO has the advantage of having oxygen functional groups such as $-\mathrm{OH},-\mathrm{COOH}$, and epoxides leading to hydrophilic features but $\mathrm{GO}$ is not compatible with organic polymers. GO is chemically modified with GOx using a polymer called $\mathrm{N}$-succinimidyl acrylate. Luo et al. designed a non-enzymatic glucose sensor based on copper nanoparticle-decorated graphene sheet (Luo et al., 2012). The introduced former researches all involve GOx, which is an enzyme, but this paper implies the probability of non-enzymatic glucose sensors. The copper nanoparticles were modified on graphene by electrodeposition. Copper electrodeposition was optimized by modulating the depositing voltage (deposition time was fixed to $400 \mathrm{~s}$ ) by comparing $0.2 \mathrm{~V}-0.55 \mathrm{~V}$. $0.5 \mathrm{~V}$ was chosen as the optimal value, and response curves were obtained having repetitive, sensitive, stable, and selective results. 
A

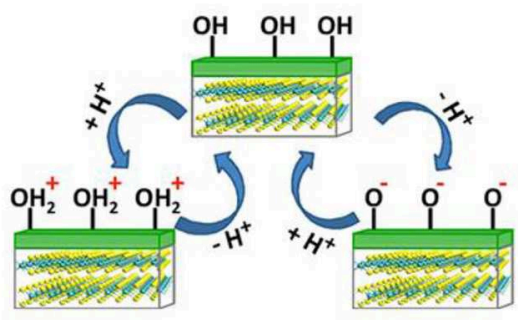

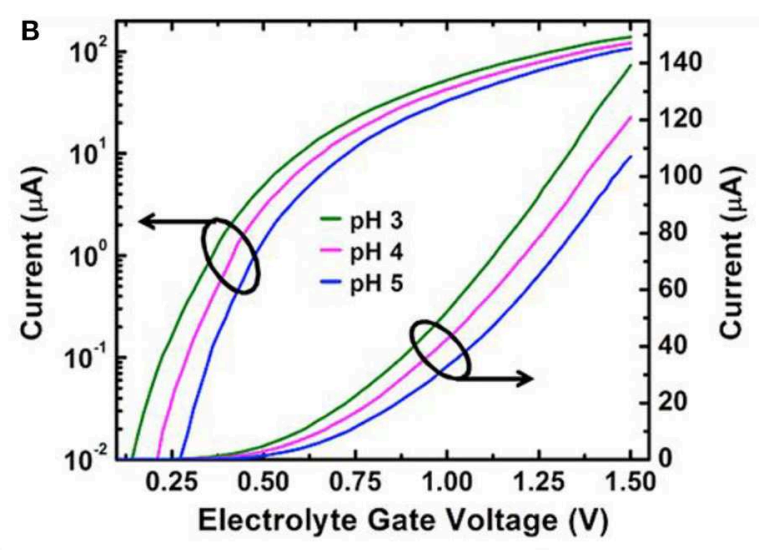

E

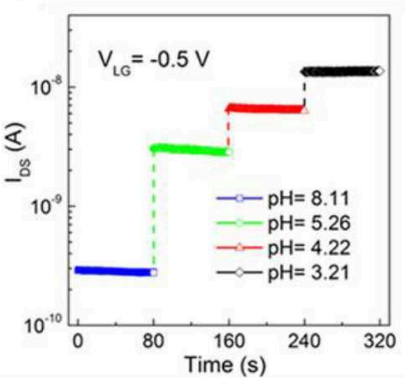

FIGURE 4 | $\mathrm{MoS}_{2}$ FET and ReS 2 FET pH sensors. (A) Sensing mechanism of $\mathrm{MoS}_{2} \mathrm{pH}$ sensors. At low pH, $\mathrm{OH}_{2}^{+}$forms by protonation of OH on the dielectric surface, consequently forming a positive surface charge on the dielectric. At high $\mathrm{pH}, \mathrm{O}^{-}$forms by deprotonation of $\mathrm{OH}$ on the dielectric surface, consequently forming a negative surface charge on the dielectric. (B) Drain current vs. electrolyte gate voltage in various pH values for an n-type MoS 2 FET. Figures (A,B) were reproduced from Sarkar et al. (2014) with permission from the American Chemical Society. (C) Illustration of ReS 2 FET for pH sensing. (D) Transfer curves (/ DS $V_{\text {LG }}$ ) at various pH values. (E) Response curves at different $\mathrm{pH}$ with $V_{\mathrm{DS}}=0.1 \mathrm{~V}$ and $V_{\mathrm{LG}}=-0.5 \mathrm{~V}$ at RT. Figures (C-E) were reproduced from Liao et al. (2018) with permission from the American Chemical Society.

\section{TMDs and Other 2D Materials}

As graphene, TMDs can also detect glucose with decorated nanoparticles. In terms of electrochemical viewpoint, nanoparticle decoration on the electrode surface not only helps electrocatalytic reactions but also presents electrochemical properties. These properties are faradic to capacitive current ratios, electron mobility, current density, and mass transport (Huang et al., 2008; Peng et al., 2009; Zeng et al., 2011). Due to these advantages, nanoparticle decoration or deposition is applied to many cases (Jeon et al., 2017). In general, there are three methods to decorate nanoparticles on surfaces including electrochemical etching, nanoband electrode fabrication, and electrodeposition of metal nanoparticles. Electrodeposition is relatively cost-effective compared to the other two methods, so the electrodeposition method is widely used. Still, electrodeposition has multiple signs of progress and may need special chemical treatments and particular conditions. Parlak et al. used $\mathrm{Au}$ nanoparticles to enhance selectivity toward glucose using a mixture of $\mathrm{MoS}_{2}$ nanosheets and $\mathrm{Au}$ nanoparticles by sonication (Parlak et al., 2017). Figure 6A indicates the schematic diagram of the $\mathrm{Au}$ nanoparticle-decorated $\mathrm{MoS}_{2}$ and proposed sensing mechanism. $\mathrm{MoS}_{2}$ nanosheets were dispersed in a $10 \mathrm{mM}$ phosphate buffer solution and were treated with ultrasonication. In Figure 6B, amperometric responses were compared between $\mathrm{MoS}_{2} / \mathrm{GOx}$ and $\mathrm{MoS}_{2} / \mathrm{Au}$ nanoparticles/GOx electrodes in $0.1 \mathrm{M}$ phosphate buffer solution and the applied voltage was $0.35 \mathrm{~V}$. $\mathrm{MoS}_{2}$-modified nanosheet electrodes had no electrocatalytic response to any concentration of glucose while $\mathrm{MoS}_{2} / \mathrm{GOx}$ and $\mathrm{MoS}_{2} / \mathrm{Au}$ nanoparticles/GOx electrodes exhibited a response. $\mathrm{Au}$ nanoparticle-doped cases have better sensitivity to glucose. $\mathrm{Su}$ et al. also utilized $\mathrm{Au}$ nanoparticles for glucose sensing enhancement. The difference between Parlak et al. and Su et al. is that Parlak et al. dispersed Au nanoparticles with ultrasonication while $\mathrm{Su}$ et al. synthesized $\mathrm{Au}$ nanoparticles onto $\mathrm{MoS}_{2}$ by the hydrothermal method (Su et al., 2014). Sensitivity and selectivity were investigated by injecting various solutions such as uric acid, dopamine, $\mathrm{NaCl}, \mathrm{KCl}$, and glucose. Only glucose had a response to the device. Huang et al. sought electrocatalytic effects from $\mathrm{Ni}$ nanoparticles rather than $\mathrm{Au}$ nanoparticles (Huang et al., 2014). Ni nanoparticles were reduced on $\mathrm{MoS}_{2}$ nanosheets by a solution process. Amperometric response with glucose, dopamine, ascorbic acid, and uric acid was investigated for response sensitivity and selectivity. Lin et al. decorated copper nano-flowers to $\mathrm{MoS}_{2}$ nanosheets. The copper was decorated using the electrodeposition process. $\mathrm{H}_{2} \mathrm{O}_{2}$ was 

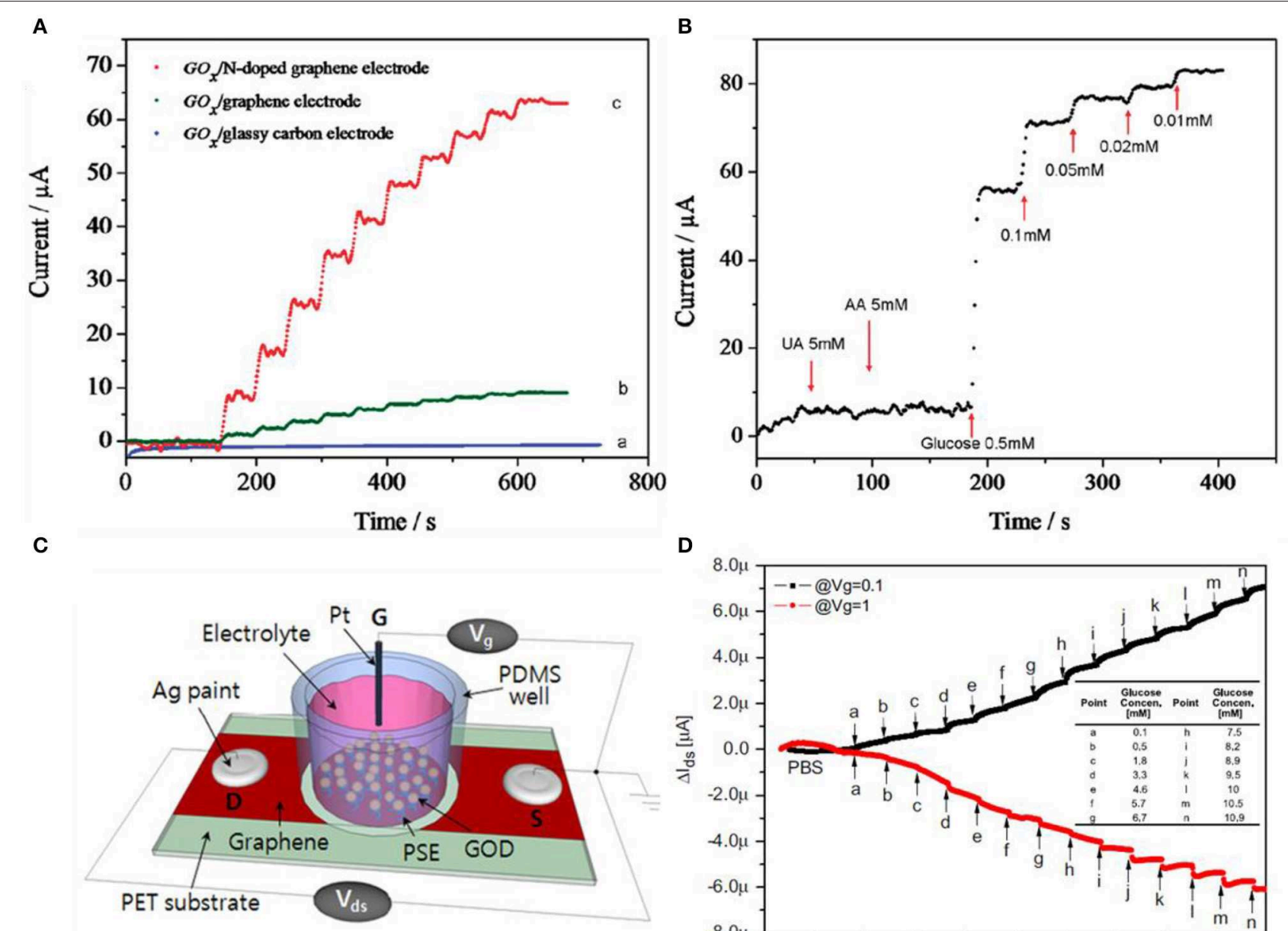

D

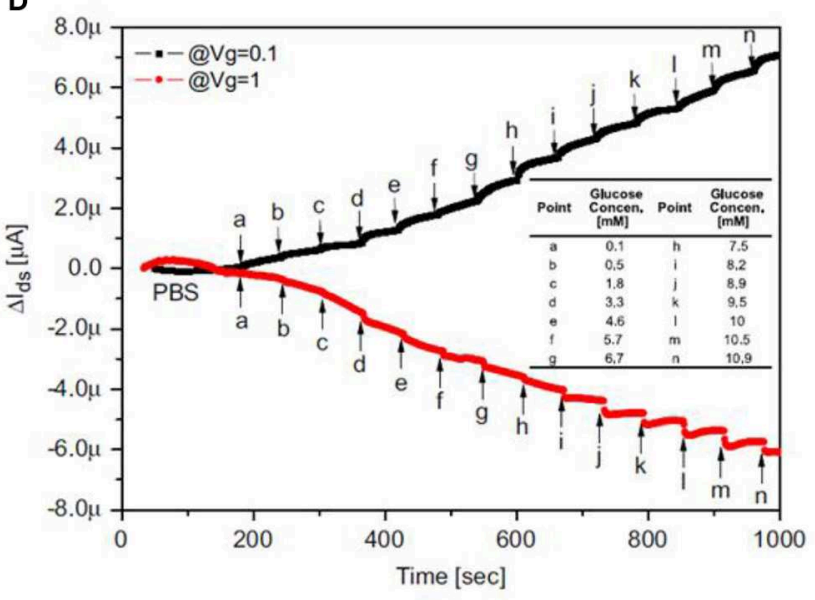

FIGURE 5 | Glucose sensors using graphene-based materials. (A) Glucose responses to various graphene-based materials. (B) N-doped graphene having selectivity to glucose than uric acid and ascorbic acid. Figures (A,B) were reproduced from Wang et al. (2010) with permission from the American Chemical Society. (C) Schematic illustrations of the CVD graphene FET sensor. (D) Response curves of glucose in different gate voltages. Source-drain voltages are fixed to $V_{D S}=-0.2 \mathrm{~V}$. Figures (C,D) were reproduced from Kwak et al. (2012) with permission from Elsevier.

selectively sensed while ascorbic acid, dopamine, fructose, lactose, and uric acid had no response (Lin et al., 2016). Cai et al. formed a $\mathrm{MoS}_{2}$-PtAg nanohybrid for colorimetric detection of glucose (Cai et al., 2016). Bimetallic nanoparticle (BNP) supported on $\mathrm{MoS}_{2}$ is much more uncommon than singlemetal nanoparticle supports due to the complexity of forming composites. Bimetallic nanoparticle decorations perform great catalytic effects due to their synergistic effect between two different metals. Recently, many groups are finding a simple method to synthesize bimetallic nanoparticle composites on $\mathrm{MoS}_{2}$ or other 2D materials (Zhong et al., 2012; Sun et al., 2015; $\mathrm{Su}$ et al., 2016) but $\mathrm{MoS}_{2}$-BNP hybrids with high quality and various heterostructures forming remain a challenge. Cai et al. prepared $\mathrm{MoS}_{2}$ with liquid exfoliation and functionalization by polyallylamine hydrochloride (PAH). After forming $\mathrm{MoS}_{2}-\mathrm{PAH}$ nanosheets, metal nanoparticles were hydrothermally reduced to the nanosheets. Two different metal precursors were prepared for Pt and Ag, respectively. Experimental results were confirmed by color change (blue) by each concentration of $\mathrm{H}_{2} \mathrm{O}_{2}$.

Not only $\mathrm{MoS}_{2}$ but also other TMDs and 2D materials are used as glucose sensors. $\mathrm{SnS}_{2}$ is an n-type semiconductor having a bandgap of $2.18-2.44 \mathrm{eV}$. They are resistive to acids and have good stability in air, which has the capability of photocatalysts. Li et al. synthesized nanoflake $\mathrm{SnS}_{2}$ shown in Figure 6C (Li et al., 2013). The left SEM image is pristine $\mathrm{SnS}_{2}$ nanoflakes and the right is GOx/MWCNTs (multiwall carbon nanotube)-SnS 2 . MWCNTs were initially mixed into nitric acid, and then after the centrifuging process, it was neutralized. These carbon nanotubes were then dispersed with yellow nanoflake $\mathrm{SnS}_{2}$ in water and vigorously mixed using ultrasonication. Then, GOx was dropped to form composites with MWCNT$\mathrm{SnS}_{2}$. After fabrication, the reproducibility and stability of the MWCNT-SnS 2 sensor are demonstrated in Figure 6D. Amperometric responses were shown and had a response to only 


\section{A}

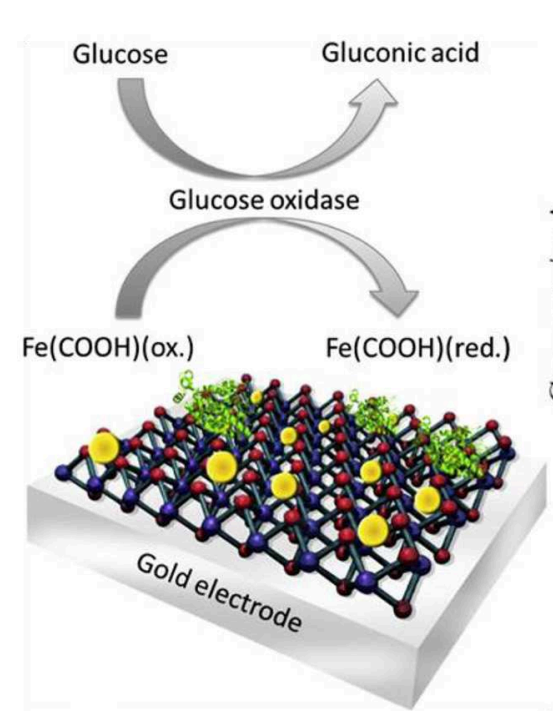

C

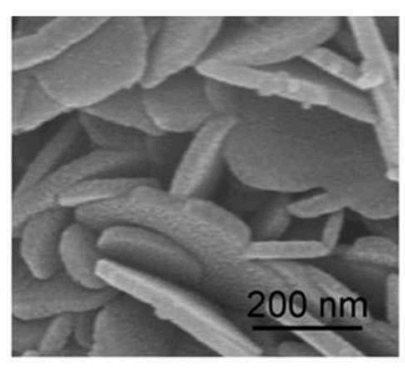

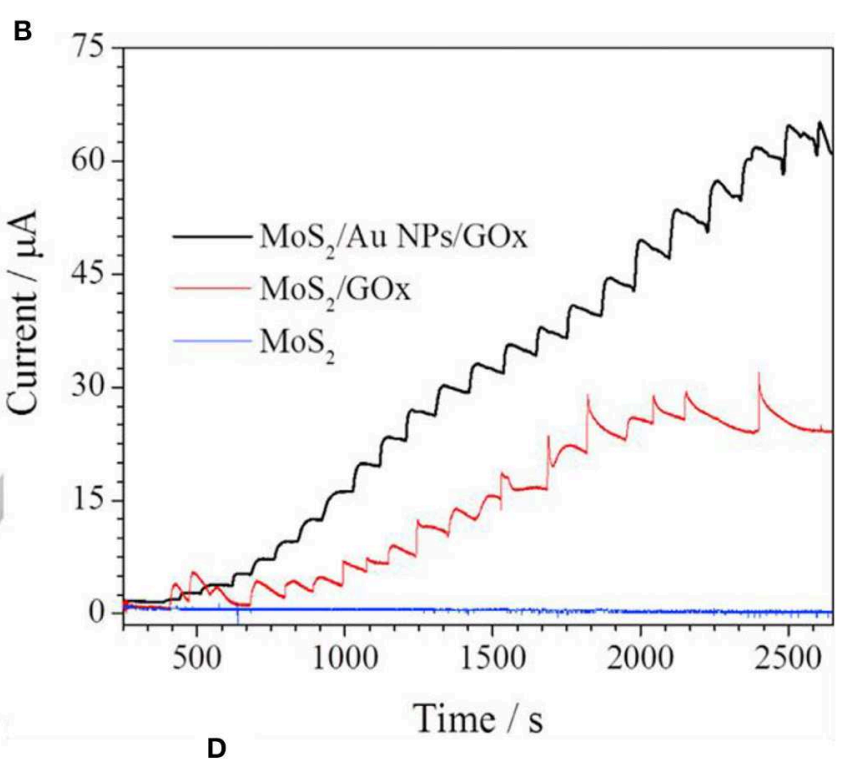

D
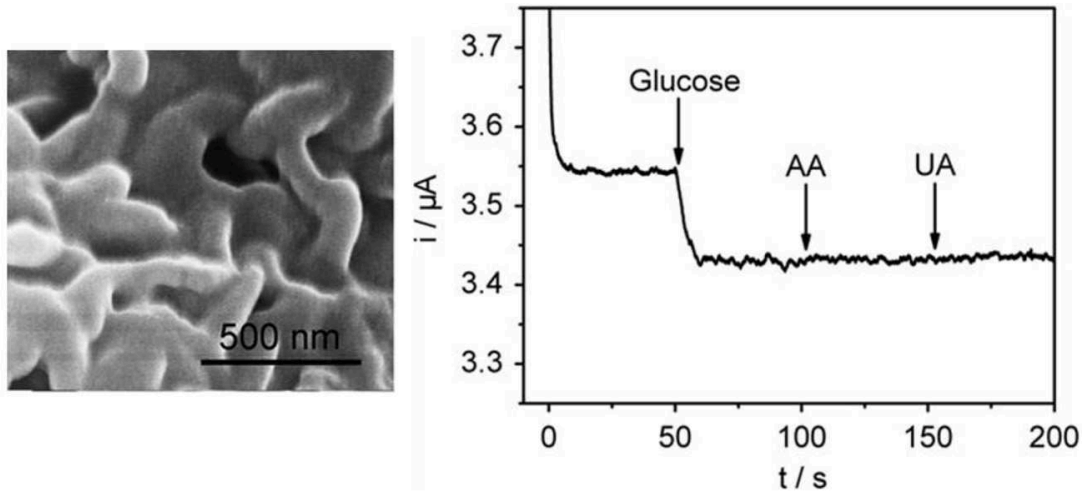

FIGURE 6 | Glucose sensors using transition metal dichalcogenides. (A) Illustration for basic glucose sensor using Au nanoparticle decorated MoS 2 . (B) Response comparison between pristine $\mathrm{MoS}_{2}, \mathrm{MoS}_{2} / \mathrm{GOx}$, and $\mathrm{MoS}_{2} / \mathrm{Au}$ NP/GOx electrodes. Figures (A,B) were reproduced from Parlak et al. (2017) with permission from Elsevier. (C) Scanning electron microscope images of pristine $\mathrm{SnS}_{2}$ (left) and GOx/MWCNTs-SnS 2 (right). (D) Response curves of glucose having selectivity at GOx/MWCNTs-SnS 2 . Figures (C,D) were reproduced from Li et al. (2013) with permission from Elsevier.

glucose while having no response to ascorbic acid and uric acid. All solutions were $0.1 \mathrm{mM}$ in $\mathrm{pH} 6.0$ phosphate buffer solution applying $-0.43 \mathrm{~V}$.

\section{METAL ION SENSING}

Due to population growth, increase in industrial and agricultural activities led to pollution in the environment and ecosystem. Recently, pollutant portable sensors have been studied in diverse ways to detect and eliminate the pollutants. The most representative pollutant from human activity is heavy metals leaking to the environment and threatening the ecosystem by inducing various diseases (Ali, 2012; Sholl and Lively, 2016). 2D materials can detect various metal ions by electrical interaction and adsorption opening a new era of water purifying (Aliprandi et al., 2017). 2D materials are single-layered and have two surfaces for adsorption having the advantage of the extremely high surface area-to-volume ratio, which leads to fast response, quick recovery rate, and multiple usages. In particular, GO can remove metal ions due to its hydrophilic feature and functional groups including oxygen atoms that can bind metal ions to the surface (Zhao et al., 2011; Sitko et al., 2013). TMDs, on the other hand, can potentially absorb certain heavy metals due to their numerous intrinsic chalcogen atoms. Especially, 2D material nanosheets integrated into FETs have recently been researched and their high sensitivity toward heavy metals was revealed. The basic sensing mechanism is the change of electrical parameters of a FET including 2D material nanosheets when the device adsorbs the typical heavy metal. These electrical parameters include field effect mobility, on current/off current ratio, and threshold voltage. It is well known that semiconducting 2D material nanosheets have high charge carrier mobility and surface energy that cause high sensitivity toward various materials. Other chemical sensors that do not involve FETs that use optical methods have the disadvantage of complicated processes or the need for chemical 
agents and long detection time. However, FETs have an extremely short detection time (real-time measurement available) by monitoring the resistance/conductance increase/decrease or the shift of charge neutrality point by the adsorption of the target substance. These devices can be miniaturized and can be developed as portable sensors supported on flexible substrates.

\section{Graphene}

Graphene may be used as the sensing material in the channel positioning in between the drain and source electrodes. The gate potential can be applied from the top or bottom, in which the former is applied to the electrical double layer in the electrode and the latter is applied through a typical thin $\mathrm{SiO}_{2}$ substrate. As the molecule/ion becomes absorbed by graphene, the local charge characteristics change and conductance is altered. Sudibya et al. fabricated reduced GO for the reasons of cost-effectiveness, solution process availability, and scalable production (Sudibya et al., 2011). Micropatterning RGO film and functionalizing it with protein give the possibility to detect diverse metal ions with high sensitivity in real time. In Figure $\mathbf{7 A}$, the fabrication process is illustrated as well as the micropatterning process of RGO on 3-aminopropyltriethoxysilane (APTES)-modified quartz. Then, the RGO thin films were functionalized with calmodulin $(\mathrm{CaM})$. Figure 7B shows the ambipolar features of RGO measured in $0.1 \mathrm{M}$ phosphate buffer saline and the inset is the scheme of overall solution-gated RGO-FET. Various metal ions were detected such as $\mathrm{Ca}^{2+}, \mathrm{Mg}^{2+}, \mathrm{Hg}^{2+}$, and so on. As shown in Figure 7C, $\mathrm{Ca}^{2+}$ ions caused conductance decline in the functionalized RGO-FET in a concentration-dependent behavior. By the difference of gate voltage, the RGO-FET has both characteristics of n-type and p-type, which is noticed by the conductance incline or decline by the injection of metal ions. The FET also bound with $\mathrm{Mg}^{2+}$, but having a lower affinity. Both ions $\left(\mathrm{Ca}^{2+}, \mathrm{Mg}^{2+}\right)$ can be bonded onto RGO by surface modification. However, $\mathrm{K}^{+}$and $\mathrm{Na}^{+}$had no response, indicating that the functional group (CaM) has no selectivity to $\mathrm{K}^{+}$and $\mathrm{Na}^{+}$. Also, without functionalization (CaM), the RGO-FET had no response to $\mathrm{Ca}^{2+}$ and $\mathrm{Mg}^{2+}$, meaning CaM is crucial for selective detection. Also, other heavy metal detection experiments were progressed by functionalizing RGO with metallothionein type II protein (MT-II), which binds with $\mathrm{Zn}, \mathrm{Cu}, \mathrm{Se}$, or $\mathrm{Cd}$, and $\mathrm{Hg}$ selectively. As shown in Figure 7D, sensing curves were depicted by the addition of $\mathrm{Hg}^{2+}$ at a detection limit of $1 \mathrm{nM}$. Comparing the detection of $\mathrm{Ca}^{2+}$ and $\mathrm{Hg}^{2+}$, mercury has much higher sensitivity than calcium, which indicates the fact that mercury has better binding affinity to MT than calcium to CaM. MTII is negatively charged in $\mathrm{pH} 7.0$, and metal binding brings MT-II closer to the RGO surface and increases the field effect from highly negative charged MT-II to RGO. As shown in Figures 7C,D, the RGO sensors are very stable in each step, having consistent conductivity. Sofue et al. detected sodium ions with a typical graphene FET (Sofue et al., 2011). Sodium ions in the electrolytes influence the electrical potential of the graphene channel. The graphene was mechanically exfoliated with adhesive tape. After exfoliating graphene on Si substrate, electrodes were formed for source and drain and a rubber frame was adhered on top of the graphene to block the leakage of electrolyte. Tris(hydroxymethyl)aminomethane and $\mathrm{HCl}$ (Tris- $\mathrm{HCl}$ ) buffer solution was used as the solution. Various concentrations of $\mathrm{NaCl}$ were added to the buffer solution to supply $\mathrm{Na}$ ions. The source drain voltage was set at $0.1 \mathrm{~V}$ and the $\mathrm{NaCl}$ concentration range was 0 to $6.0 \mathrm{mM}$. Sodium ions adsorb/desorb the hydroxyl groups from the graphene surface. $\mathrm{As} \mathrm{Na}^{+}$ions increase on the graphene surface by the addition of $\mathrm{NaCl}$ solution, the positive charge accumulates on the graphene channel. On the other hand, when $\mathrm{Na}^{+}$ion concentration is low, these ions adsorb to the hydroxyl group. Consequently, the charge neutrality point shifts toward the negative direction as the concentration increases. Transfer curves in diverse $\mathrm{Na}^{+}$concentrations were plotted and curve shifting happened. Also, the current-time graph was plotted and the drain current decreased as $\mathrm{Na}$ ion concentration increased. Wen et al. detected lead ions using Au nanoparticles and DNAzyme functionalization onto graphene (Wen et al., 2013). Au nanoparticles were decorated initially on graphene due to DNAzyme molecule immobilization. DNAzymes have the advantage of low cost, high stability, and selectivity compared to protein and RNA enzymes. Many DNAzyme molecules adhere to a single $\mathrm{Au}$ nanoparticle. Graphene transfer curves shift positively in a large order when decorated with Au nanoparticles (reduced from $\mathrm{HAuCl}_{4}$ ) due to p-type doping effect. DNA molecule induces $n$-doping effects to graphene and curves shift to the negative direction. Consequently, the DNAzyme/Au nanoparticles/graphene curve is in the intermediate of graphene and $\mathrm{Au}$ nanoparticles/graphene curves. When lead ions become adjacent to the device, the transfer curves shift to the right side again and the shifting is proportional to the concentration of lead.

\section{TMDs and Other 2D Materials}

TMDs also have high binding energy to heavy metal ions due to their chalcogenide ions. Jiang et al. used $\mathrm{MoS}_{2}$-based FET to sense mercury ions. $\mathrm{MoS}_{2}$ has a high affinity to $\mathrm{Hg}^{2+}$ due to their sulfur sites that are bonded extremely strong (Jiang et al., 2015). Binding with mercury ions leads to a p-type doping effect and eventually reduces the electron concentration. This affects the electrical and optical features of $\mathrm{MoS}_{2}$ such as electron transport and photoluminescence properties. The sensitivity of the device is confirmed by monitoring the conductance change when mercury ions are introduced. As shown in Figure 8A, flakes of $\mathrm{MoS}_{2}$ were mechanically exfoliated to a $\mathrm{Si} / \mathrm{SiO}_{2}$ substrate, and then electron-beam lithography and electronbeam evaporation were used to define contact electrodes. After the process, a polydimethylsiloxane (PDMS) microfluidic channel was introduced to the device for $\mathrm{Hg}^{2+}$ solution delivery. The $\mathrm{Hg}^{2+}$ solution was made by dissolving $\mathrm{Hg}\left(\mathrm{ClO}_{4}\right)_{2}$ in DI water. In Figure 8B, to check the sensing properties of the device, $\mathrm{Hg}^{2+}$ solutions possessing various concentrations flowed into the device. As the concentration increased, the conductance declined due to charge impurity-induced scattering effects. As $\mathrm{Hg}^{2+}$ increases, the p-type doping effect also increases (transfer curves shift to the right as a higher concentration of $\mathrm{Hg}^{2+}$ is introduced), leading to electrons to decrease, resulting in the reduction of carrier mobility. Also, in Figure $\mathbf{8 C}$, selectivity 


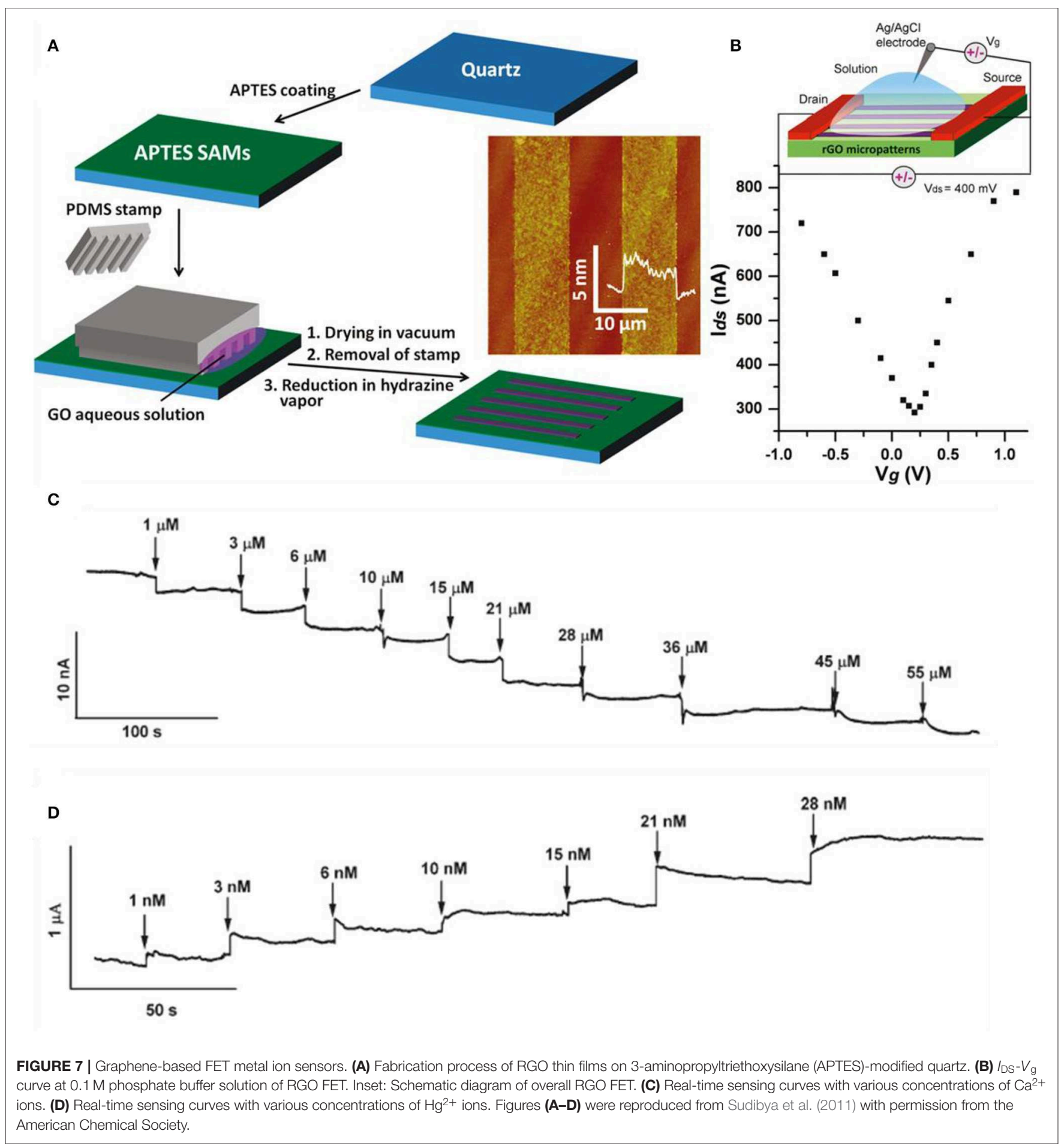

was confirmed by testing with various heavy metal ions whose concentrations were all $1 \mathrm{nM}$. Zhou et al. also detected $\mathrm{Hg}^{2+}$ with $\mathrm{MoS}_{2}$ by decorating specific DNA functional groups. Figure 8D indicates the fabrication process of the $\mathrm{MoS}_{2} / \mathrm{DNA}$ Au nanoparticle hybrid structure. $\mathrm{MoS}_{2}$ nanosheets were formed by $\mathrm{Li}$ ion exfoliation at RT. $\mathrm{MoS}_{2}$ powder was intercalated by lithium ions and then they were sonicated in water. $\mathrm{MoS}_{2}$ crystals were formed and then immersed into a butyllithium solution for a week filled with $\mathrm{Ar}$ gas. $\mathrm{Li}_{\mathrm{x}} \mathrm{MoS}_{2}$ was retrieved by centrifugation and was washed to eliminate the residues. Ultrasonication was processed for exfoliation to form nanosheets. Then, the film was transferred onto a $\mathrm{SiO}_{2}$ substrate as shown in Figure 8D. Au nanoparticles were deposited on the film by sputtering, resulting in 2-nm decoration on the surface. Then, 
the DNA solution was injected on the active site of the device and was rinsed with deionized water to eliminate redundant DNA. When $\mathrm{Hg}^{2+}$ is introduced to the device, $\mathrm{T}-\left(\mathrm{Hg}^{2+}\right)-\mathrm{T}$ chelates, formed by the reactions between $\mathrm{Hg}^{2+}$ and the thymidine of the DNA molecules resulting in electrical properties to change $\mathrm{MoS}_{2}$. Real-time detections of $\mathrm{Hg}^{2+}$ in water are illustrated in Figure 8E. The black curve indicates the platform of $\mathrm{MoS}_{2} / \mathrm{DNA}-$ $\mathrm{Au}$ nanoparticles, the purple dash curve indicates $\mathrm{MoS}_{2}-\mathrm{Au}$ nanoparticles, and the blue dash curves indicate $\mathrm{MoS}_{2}$ only. From the curves in Figure 8E, it is noticed that the $\mathrm{MoS}_{2}$ has higher conductivity as $\mathrm{Hg}^{2+}$ concentration increases. The results are opposite from Jiang et al., which explains that $\mathrm{MoS}_{2}$ from Zhou et al. is a p-type semiconductor. From the fabricating process, $\mathrm{MoS}_{2}$ was exposed to an oxygen-containing atmosphere and the oxygen molecules were adsorbed onto the defects or sulfur sites on the layer of $\mathrm{MoS}_{2}$ film and eventually worked as p-type dopants by trapping electrons (Zhou et al., 2016).

Li et al. fabricated thin-layered black phosphorus (BP) $\mathrm{Hg}^{2+}$ sensors by mechanically exfoliating $\mathrm{BP}$ with adhesive tape and transferring it to the $\mathrm{Si} / \mathrm{SiO}_{2}$ substrate. The source, drain, and the gate was defined by photolithography and then sputtered with $\mathrm{Cr}$ and $\mathrm{Au}$. The device was introduced to $0.1 \mathrm{M}$ sodium acetate buffer solution with $\mathrm{pH}=4.6$ with various $\mathrm{Hg}^{2+}$ concentrations from 0.01 to $100 \mathrm{ppb}$ at RT. The concentration increases of $\mathrm{Hg}^{2+}$ led to the transfer curves to reduce in current, suggesting an increase of resistivity of the BP channel. BP is a typical p-type semiconductor, which means holes are the main charge carriers. When $\mathrm{Hg}^{2+}$ ions are attached to the surface of $\mathrm{BP}$, they act as a positive gate voltage. In other words, $\mathrm{Hg}^{2+}$ ions repel positively charged holes. Consequently, the Fermi energy shifts from the valence band (i.e., charge neutrality point shifts to the negative direction) and hole density reduces, resulting in $\mathrm{BP}$ conductivity decline ( $\mathrm{Li}$ et al., 2017).

\section{OTHER CHEMICAL SENSING}

\section{Dopamine, Ascorbic Acid, and Uric Acid Sensing With Various 2D Materials}

Dopamine (DA), ascorbic acid (AA), and uric acid (UA) are very essential to human metabolism because they influence the central nervous system and serum by coexisting. The lack or excess of DA, AA, and UA may lead to various disorders that can be fatal to life. The importance of direct, simultaneous, quick sensing of DA, AA, and UA cannot be emphasized. Overall, 2D material-based DA, AA, and UA sensors accompany doping, metal nanoparticle decoration, and reduction process to enhance selectivity and sensitivity. He et al. manufactured a dopamine device based on RGO FET by reducing patterned GO films. Figure 9A shows the schematic diagram of the experimental setup of the top-gate (solution gated) RGO FET for dopamine sensing. After patterning GO with the capillary method, the chemical reduction process was followed by hydrazine vapor. Before micropatterning, the $\mathrm{SiO}_{2}$ substrate was modified with 3-aminopropyltriethoxysilane (APTES) to supply a positively charged surface. This process provides the uniformity of GO patterns and prevents aggregation on the substrate. Figure 9B shows the detection of dopamine in the RGO FET device at gate voltages $V_{\mathrm{g}}=0.6 \mathrm{~V},-0.6 \mathrm{~V}$. Each step indicates the addition of dopamine increasing the concentration ranging from 1 to $8 \mathrm{mM}$. Dopamine can easily bind with RGO through strong $\pi-\pi$ interactions. Also, the transfer curves shifted to the right, showing that they induce p-doping or incline the hole concentration in the RGO film (He et al., 2010). Yang et al. demonstrated a typical RGO sensor for simultaneous detection of dopamine, ascorbic acid, and uric acid. The GO sheet was synthesized by the Hummers method and they were electrochemically reduced. The experiments were done with cyclic voltammograms. Each peak current is proportional to the concentration of each solution. The detection limits for $\mathrm{DA}, \mathrm{UA}$, and $\mathrm{AA}$ are $0.5,0.5$, and $250 \mu \mathrm{M}$, respectively. Moreover, not only measuring with DA, UA, and AA but also mixing with other inorganic ions such as 100 -fold $\mathrm{K}^{+}$, $\mathrm{Ca}^{2+}, \mathrm{Na}^{+}, \mathrm{Mg}^{2+}, \mathrm{Zn}^{2+}, \mathrm{NH}_{4}^{+}, \mathrm{Cl}^{-}, \mathrm{SO}_{4}^{2-}, \mathrm{NO}_{3}^{-}$, and $\mathrm{HCO}_{3}^{-}$ do not interfere with the main peaks, indicating that the sensor has great selectivity toward DA, UA, and AA (Yang et al., 2014). Other groups used graphene doping Pt to form nanocomposites (Sun et al., 2011) or doping nitrogen (Sheng et al., 2012). Yang et al. formed graphene/Pt nanoparticle composites by dissolving $\mathrm{PtCl}_{4}$ in ethylene glycol containing $0.1 \mathrm{M} \mathrm{NaOH}$. After vigorously stirring, graphene powders were added to the $\mathrm{Pt}$ colloidal solution and then the solution was ultrasonicated. After filtration and drying process, the $\mathrm{Pt}$ /graphene catalyst was obtained. The experiments were done using cyclic voltammograms comparing bare glassy carbon (GC), graphene, and graphene/Pt nanocomposite electrodes. Anodic peak currents were $102 \mu \mathrm{A}$ (GC), $183 \mu \mathrm{A}$ (graphene), and $371 \mu \mathrm{A}$ (graphene/Pt). From these results, both graphene and graphene/Pt-modified electrodes increase the electrochemical sensitivity. The amperometric responses of AA, DA, and UA were measured. All experiments were progressed in a $0.1 \mathrm{M}$ phosphate buffer solution at $0.5 \mathrm{~V}$ and the detection limits were $0.15 \mu \mathrm{M}$ (AA), $0.03 \mu \mathrm{M}$ (DA), and $0.05 \mu \mathrm{M}$ (UA). Sheng et al. doped nitrogen on graphene for the simultaneous determination of AA, DA, and UA. After mixing GO and melamine, the mixture was annealed to $800^{\circ} \mathrm{C}$ for $1 \mathrm{~h}$ in $\mathrm{Ar}$ atmosphere. Then, the final product was taken out from the tube. The electrochemistry of each solution was measured. All three cases had excellent electrocatalytic activity toward the oxidation of AA, DA, and UA. The interactions between the target biomolecules (hydrogen bonds), nitrogen-doped graphene $(\pi$ $\pi$ stack), structure of the molecules, and the unique structural and properties of nitrogen-doped graphene influenced the electrocatalytic activity.

Sun et al. decorated $\mathrm{Au}$ nanoparticles onto $\mathrm{MoS}_{2}$ nanosheets for the detection of $\mathrm{AA}, \mathrm{DA}$, and UA. In Figure 9C, the overall characterization, fabrication, and electrochemical sensing process are illustrated. The $\mathrm{MoS}_{2}$ nanosheets were prepared by the intercalation exfoliation method. The Au nanoparticles were decorated by the electrochemical deposition method. The synergetic effects of $\mathrm{MoS}_{2}$ and $\mathrm{Au}$ nanoparticles enhanced the oxidation reactions of $\mathrm{AA}, \mathrm{DA}$, and UA. Figures $9 \mathrm{D}, \mathrm{E}$ are 
A

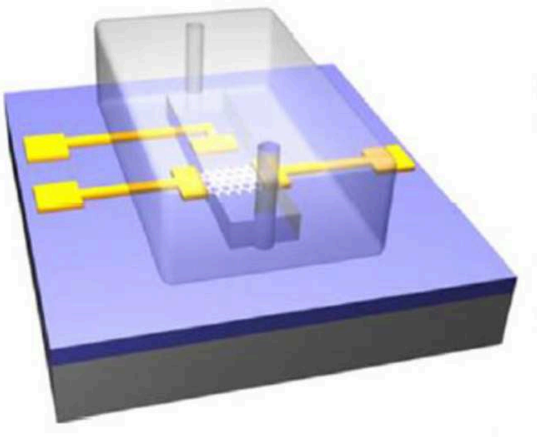

C

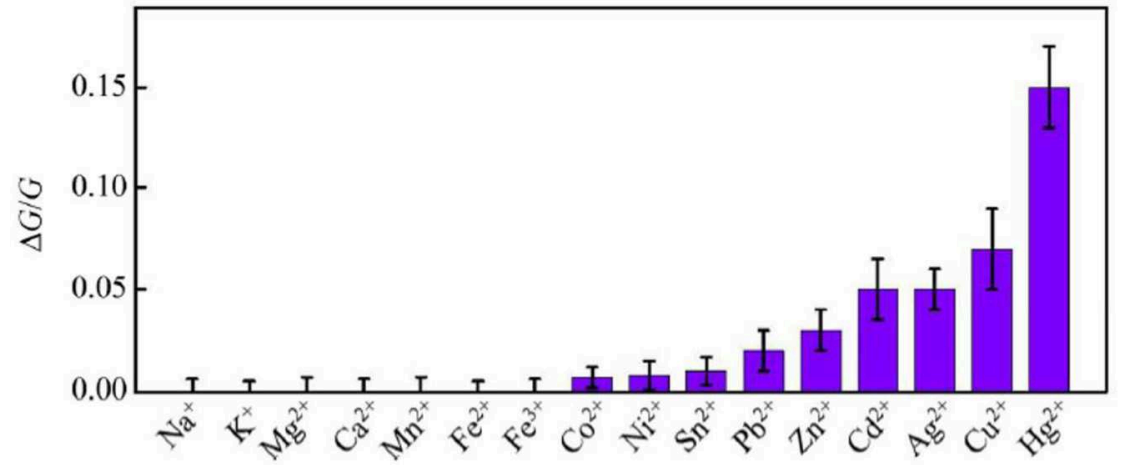

D

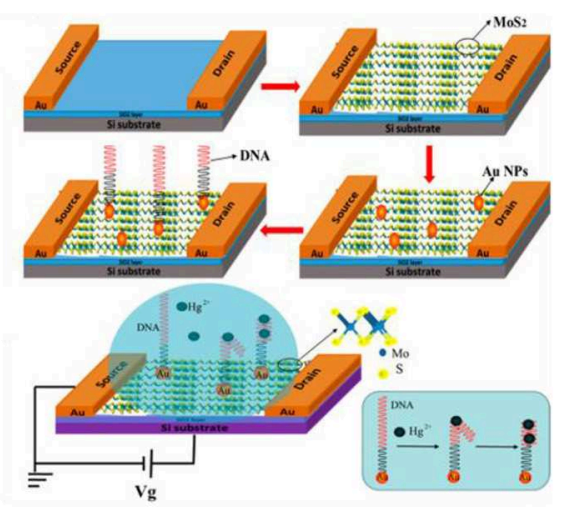

B

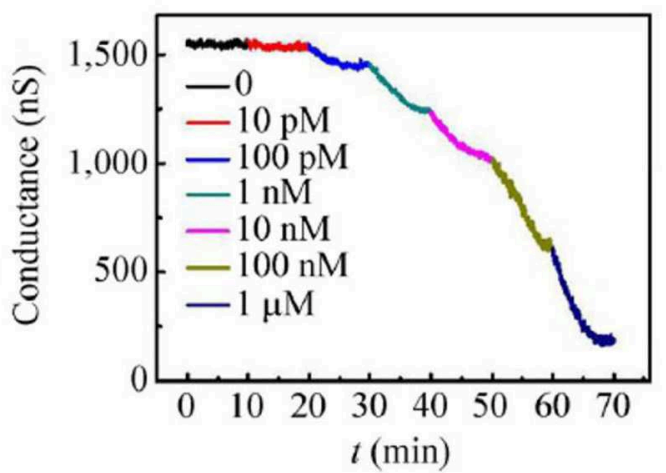

E

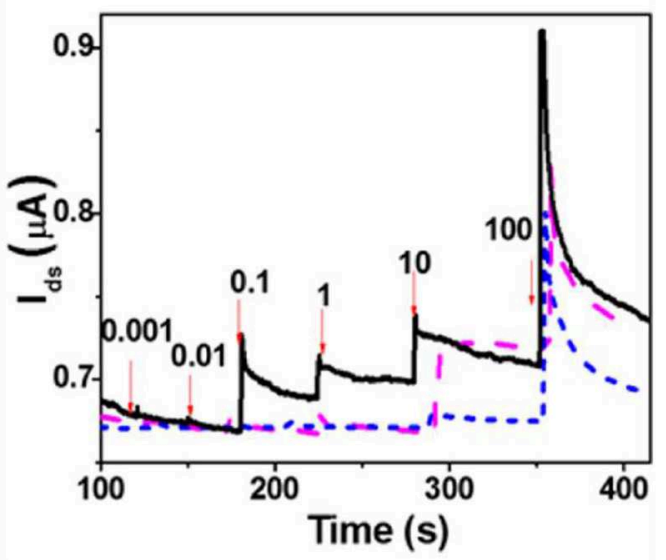

FIGURE 8 | MoS 2 -based FET metal ion sensors. (A) Schematic diagram of microfluidic channel onto $\mathrm{MoS}_{2}$ device. (B) Sensing curve data using low-concentration $\mathrm{Hg}^{2+}$ ions. (C) Selectivity to various metal ions having a concentration of $1 \mathrm{nM}$. Figures (A-C) were reproduced from Jiang et al. (2015) with permission from Springer. (D) Illustration of manufacturing process of $\mathrm{MoS}_{2}$ based FET. MoS2/DNA-Au NPs hybrid structure is shown. (E) Real-time sensing curves of $\mathrm{Hg}^{2+}$ ( $\mathrm{nM}$ ) in water ( $V_{\mathrm{DS}}$ $=0.1 \mathrm{~V}$ ), $\mathrm{MoS}_{2} / \mathrm{DNA}-\mathrm{Au} \mathrm{NPs}$ (black, solid), MoS 2 -Au NPs (purple, dash), $\mathrm{MoS}_{2}$ (blue, short dash). Figures (D,E) were reproduced from Zhou et al. (2016) with permission from the American Chemical Society.

scanning electron microscopy images of pristine $\mathrm{MoS}_{2}$ and $\mathrm{Au}$ nanoparticle-decorated $\mathrm{MoS}_{2}$ nanostructures on ITO. The detection limits for AA, DA, and UA were 100, 0.05, and $10 \mu \mathrm{M}$, respectively. Wu et al. electrochemically reduced $\mathrm{MoS}_{2}$ singlelayered nanosheets to detect various biomolecules such as DA in the presence of AA and UA. The oxidation peak of AA was much lower than that of DA or UA. The DA oxidation signal being stronger than AA or UA is due to the negative charges existing on reduced $\mathrm{MoS}_{2}$, which repel the anionic AA and UA but attract the cationic DA. The peak current of DA increased linearly with the DA concentration from 1 to $50 \mu \mathrm{M}$ (Wu et al., 2012). 
A Ag/AgCl electrode

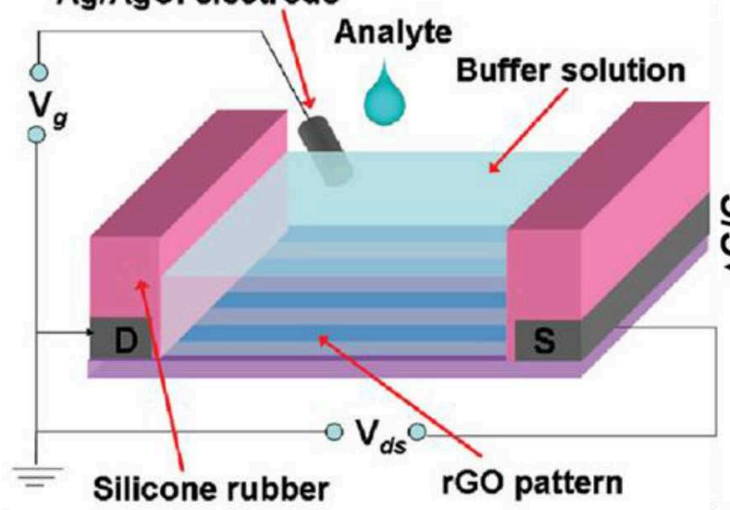

B

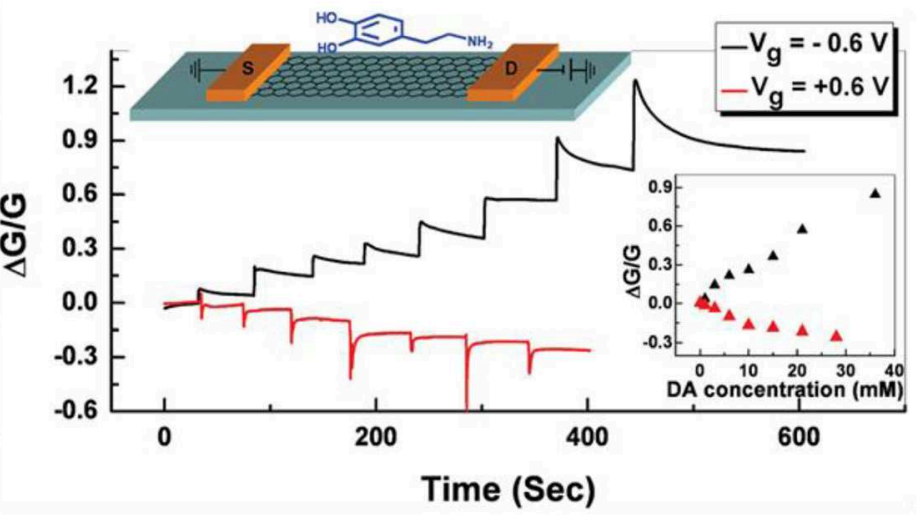

AA DA UA

C

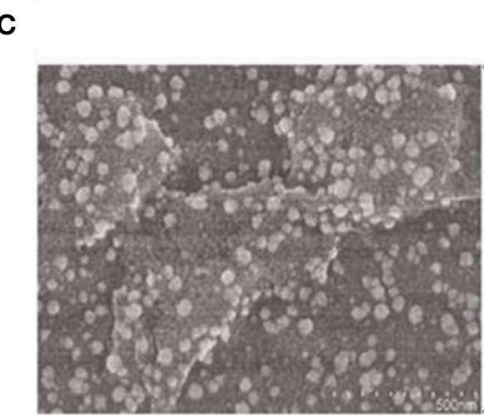

D

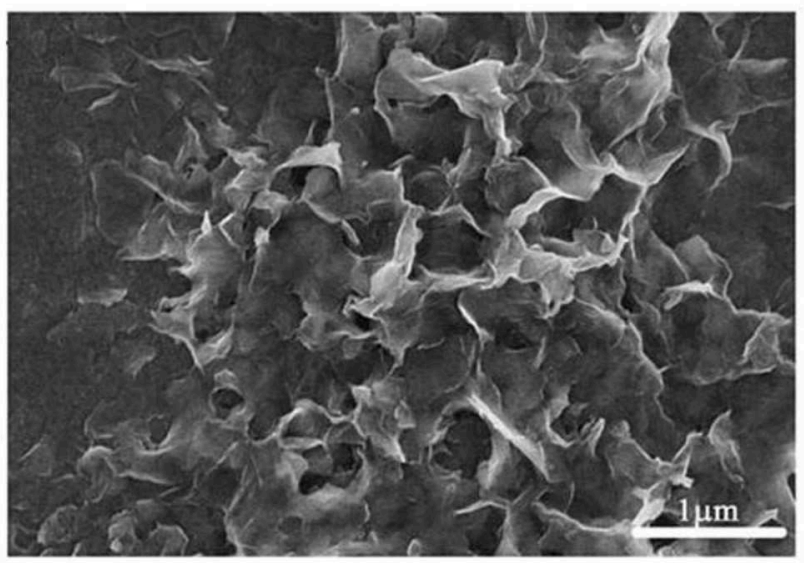

E

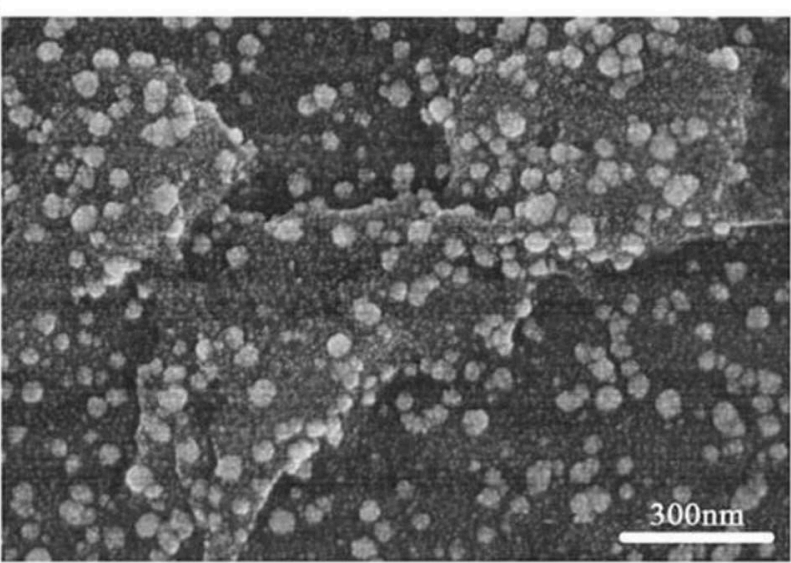

FIGURE 9 | Dopamine, uric acid, and ascorbic acid sensors using 2D materials. (A) Schematic figure of RGO FET for dopamine sensing. (B) Detection curves of RGO FET at $V_{g}=0.6 \mathrm{~V}$; each step indicates the gradual addition of dopamine ranging from 1 to $8 \mathrm{mM}$. Figures (A,B) were reproduced from He et al. (2010) with permission from the American Chemical Society. (C) Schematic illustrations of gold-decorated $\mathrm{MoS}_{2}$-based electrochemical sensors. (D) Scanning electron microscopy images of pristine $\mathrm{MoS}_{2}$ and (E) gold nanoparticle-decorated $\mathrm{MoS}_{2}$. Figures (C-E) were reproduced from Sun et al. (2014) with permission from the Royal Society of Chemistry.

\section{Nucleic Acid Sensing With Various 2D Materials}

Biomolecule detecting and identifying in a solution is gaining importance these days due to the use of various areas such as medical diagnostics, food analysis, and state-of-the-art technology and science. Existing techniques of biomolecule sensing were based on fluorophores or enzymes, which have great sensitivity but cannot be monitored in real time; in other words, the response takes a long time. Also, the cost and complexity of monitoring for biological agents are high. To reduce the complexity of traditional biomolecule sensors, many types of researches and studies were based on detecting the target molecules by binding directly to the sensor. Surface plasmon resonance (SPR) was emerging for label-free biological detection 

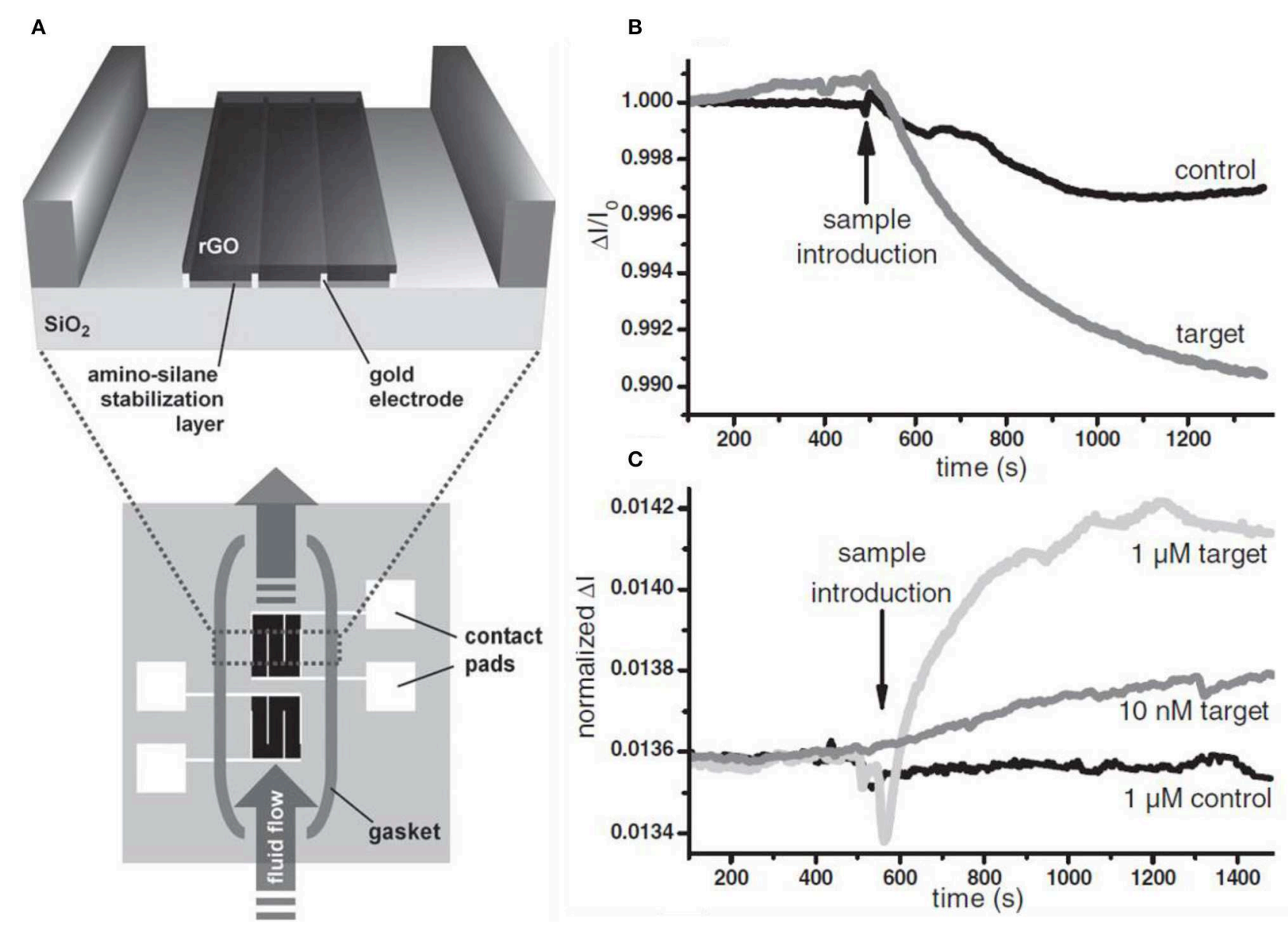

D

E
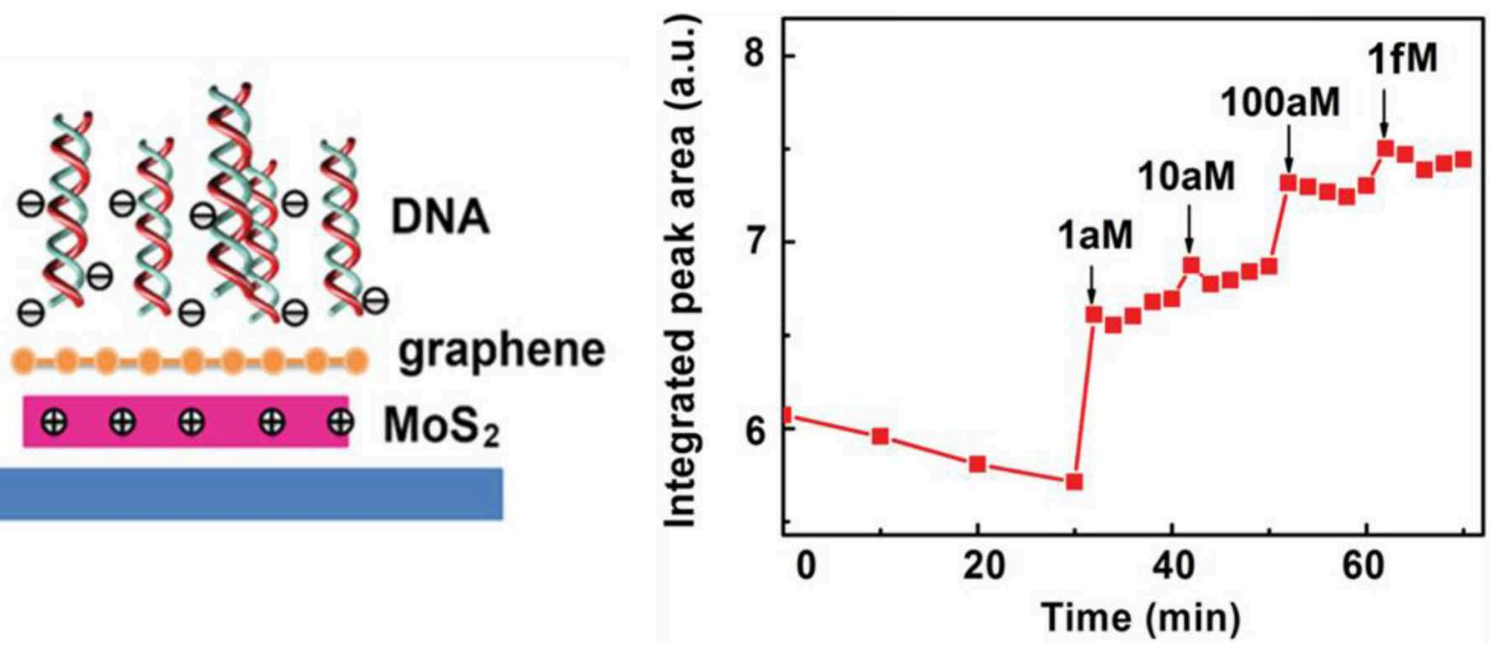

FIGURE 10 | RGO FET for real-time DNA detection. (A) Schematic diagram of RGO FET. RGO is deposited on top of the pre-fabricated electrodes. The two devices are isolated, to eliminate non-specific biological adhesion (bottom), which acts as a reference device. (B) RGO FET sensing curves toward DNA. Non-specific binding (control curve) occurs to the first device (bottom device in (A). (C) Second device curves having much more selectivity toward the target DNA. Figures (A-C) were reproduced from Stine et al. (2010) with permission from Wiley. Graphene/MoS 2 heterostructures for detection of DNA. (D) Scheme of the charge distribution when DNA becomes adjacent. (E) Photoluminescence response of graphene/MoS 2 to increased concentration of the target DNA. Figures $(\mathbf{D}, \mathbf{E})$ were reproduced from Loan et al. (2014) with permission from Wiley. 


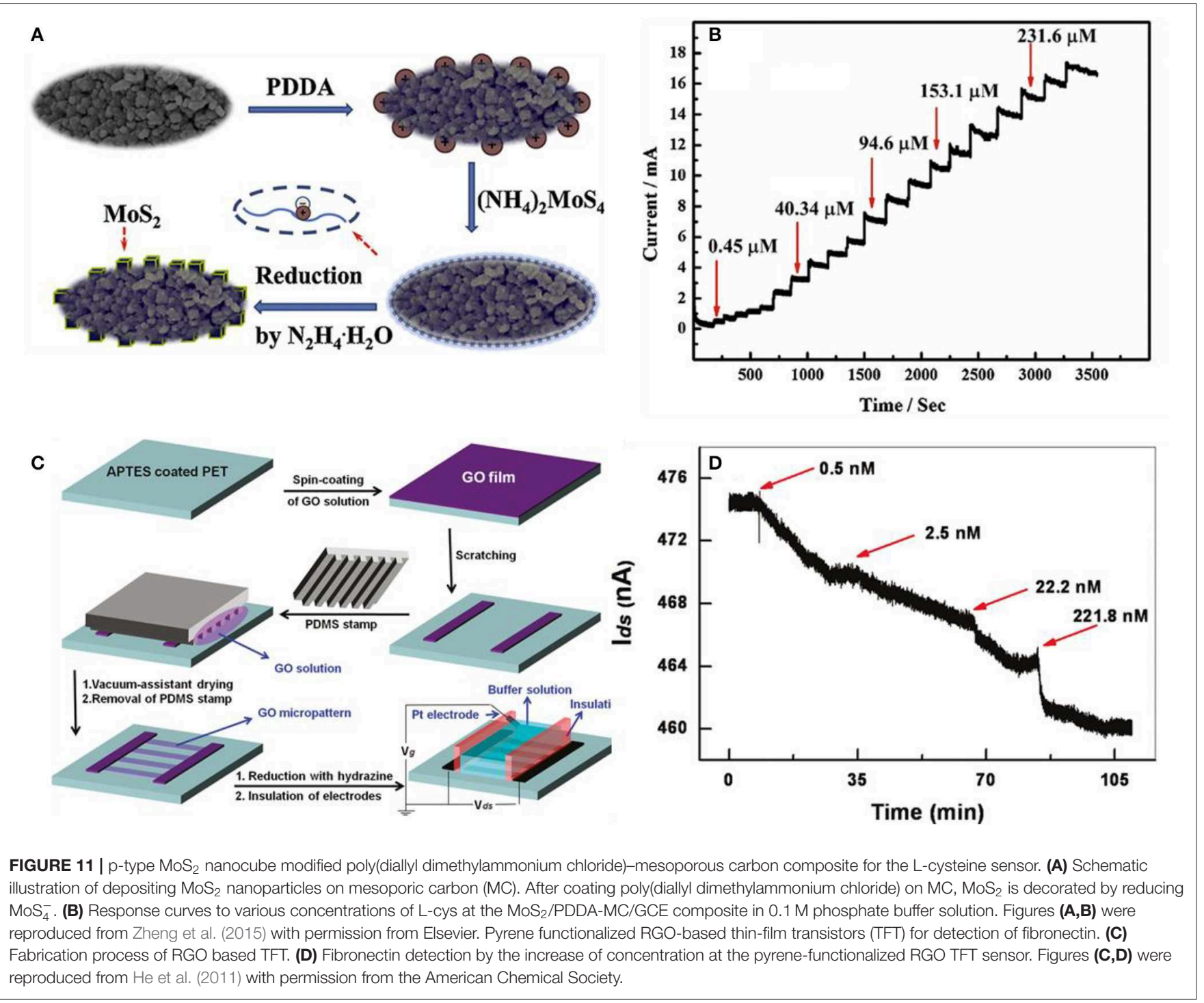

(Homola, 2008). Recently, biomolecule sensors based on silicon nanowire (Curreli et al., 2008; Stern et al., 2008) and carbon nanotube (Allen et al., 2007) FETs have higher sensitivities. These enhanced nanoscale sensors are due to their high surface area-tovolume ratios; thus, only a small number of charged molecules are needed to change the electrical characteristics of the FET (Cui et al., 2001). These nanowire/tube devices have difficulties with alignment and reproducibility issues. Recently, using 2D materials such as graphene, GO, RGO, and TMDs is emerging due to easy fabrication (exfoliation process, functionalization), low cost, and high sensitivity. In particular, GO has oxygen group functions and defects that bond selectively with biomolecules and they are hydrophilic. RGO also has functional groups and high conductivity. Stine et al. deposited RGO onto the $\mathrm{SiO}_{2}$ substrate and detected DNA in real time. Figure 10A shows the schematic diagram of the RGO FET. The device is fabricated into two devices, which are isolated inside the gasket. The bottom device acts as a reference to eliminate non-specific DNA adhesion. The magnified area is the deposition of GO on top of the prefabricated electrodes. The GO is reduced by hydrazine exposure. Figure 10B shows the single FET device having no specificity to the target DNA. The target DNA (complementary DNA) has more conductance difference than the controlled specimen (noncomplementary DNA) but the sensor clearly responded to both cases. Using the second FET solved the selectivity problem as plotted in Figure 10C. One device was activated as an internal reference, and there was no response to the control sample. The existence of reference led to selectivity toward the target DNA, and also non-complex functionalization was needed (Stine et al., 2010). Yin et al. demonstrated decorating Pt nanoparticles onto a conductive RGO thin film by photochemical reduction. The DNA detection was based on the Pt-S covalent bonding and immobilizing the DNA. The transfer curves shifted to the negative direction, meaning the DNA caused n-doping effects. 


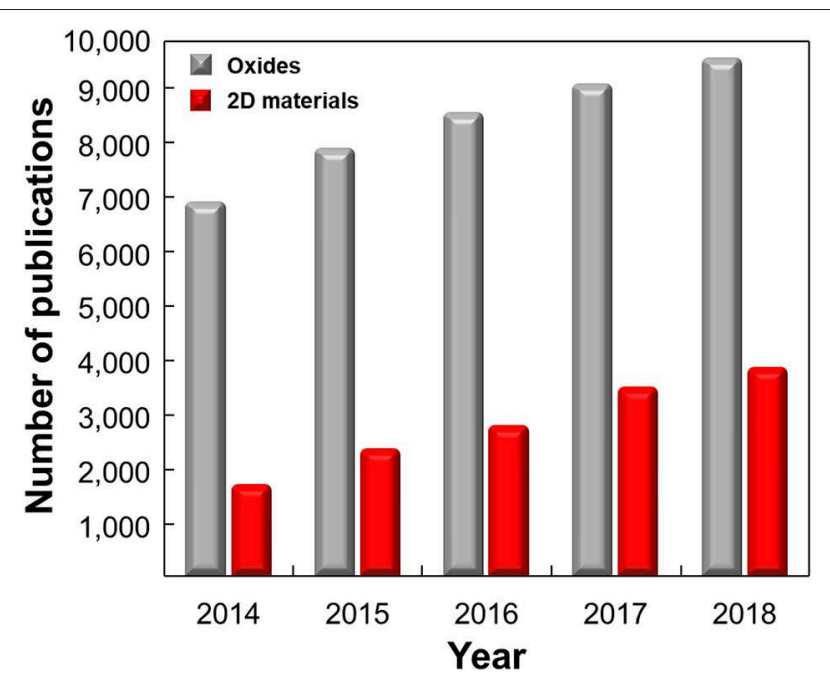

FIGURE 12 | Number of publications on ion/molecule sensors based on 2D materials and metal oxides by years. The data were collected using the Web of Science [v. 5. 32]-Web of Science Core Collection Search: keywords of ((")hydrogen ion" OR glucose OR $\mathrm{Pd}^{2+} \mathrm{OR} \mathrm{Sr}^{2+} \mathrm{OR} \mathrm{Cu}^{2+} \mathrm{OR} \mathrm{Cd}^{2+} \mathrm{OR} \mathrm{Hg}^{2+}$ OR $\mathrm{Pb}^{2+} \mathrm{OR} \mathrm{Cr}^{4+}$ OR $\mathrm{Ni}^{2+}$ OR $\mathrm{Co}^{2+} \mathrm{OR} \mathrm{Mn}^{2+}$ OR uric OR ascorbic OR dopamine OR DNA OR amino OR protein sens ${ }^{*}$ ) AND (graphene OR MoS $\mathrm{OR}_{2} \mathrm{OR}$ molybdenum disulfide OR black phosphorous OR $\mathrm{MoSe}_{2} \mathrm{OR}$ molybdenum selenide $\mathrm{OR} \mathrm{WS}_{2}$ OR tungsten disulfide OR WSe $\mathrm{WR}_{2}$ OR tungsten diselenide)) and (“"hydrogen ion" OR glucose OR $\mathrm{Pd}^{2+} \mathrm{OR} \mathrm{Sr}^{2+} \mathrm{OR} \mathrm{Cu}^{2+} \mathrm{OR} \mathrm{Cd}^{2+} \mathrm{OR}$ $\mathrm{Hg}^{2+} \mathrm{OR} \mathrm{Pb}^{2+} \mathrm{OR} \mathrm{Cr}^{4+} \mathrm{OR} \mathrm{Ni}^{2+} \mathrm{OR} \mathrm{Co}^{2+} \mathrm{OR} \mathrm{Mn}^{2+}$ OR uric OR ascorbic OR dopamine OR DNA OR amino OR protein sens ${ }^{\star}$ ) AND (oxide)) were used.

Due to the selective effect of Pt nanoparticles, the shift of Pt nanoparticles/RGO was much larger than pristine RGO. The DNA detection limit of real-time measurements was $2.4 \mathrm{nM}$ (Yin et al., 2012).

Loan et al. designed a heterostructural structure by stacking graphene on $\mathrm{MoS}_{2}$, providing ultrasensitivity to DNA. The role of graphene is to protect the reaction between $\mathrm{MoS}_{2}$ and the surrounding environment and to be a biocompatible superficial layer to immobilize DNA molecules on its surface. The photoluminescence intensity of the $\mathrm{MoS}_{2}$ from the stack of graphene/ $\mathrm{MoS}_{2}$ increased as the concentration of DNA increased by adding them. Real-time measurements were available in a few minutes and the detection limit was reached to the level of aM. In Figure 10D, the schematic of the heterostructure is depicted. When DNA approaches the heterostructure, the DNA adsorption on the composite results in more positive charges in the $\mathrm{MoS}_{2}$, which enhances its PL intensity. Also, graphene is not affected by the DNA sensing by confirming the Raman spectra, suggesting that the Fermi energy of graphene is not changed by DNA adhering. This is due to the electron transfer of $\mathrm{MoS}_{2}$ to graphene, which means any induced hole of graphene is filled by the electron from $\mathrm{MoS}_{2}$, resulting in $\mathrm{MoS}_{2}$ becoming reduced due to the loss of electrons. Figure 10E is the plot of $\mathrm{PL}$ experiments having greater intensity as the concentration of DNA is increased (Loan et al., 2014).

\section{Amino Acid and Protein Sensing With Various 2D Materials}

Protein is the basic unit that makes up any living organism. Protein and amino acid detection are crucial in medical diagnosis (Yoo et al., 2014), food security (Setford et al., 2002), and biotechnology (Zhang et al., 2016). These molecules are also compatible with $2 \mathrm{D}$ materials as nucleic acids. However, amino acid and protein are much larger in molecular weight and size than nucleic acids. L-cysteine (L-Cys) is an example of a crucial amino acid, which plays a vital role biologically. This amino acid can be used as an indicator for several diseases (Shahrokhian, 2001), and L-Cys has been applied in industrial purposes such as cosmetics, food procedure, and pharmacy uses (Lima et al., 2008). Zheng et al. designed $\mathrm{MoS}_{2}$-modified poly(diallyl dimethyl ammonium chloride) (PDDA)-mesoporous carbon (MC) composites to detect L-Cys. The fabrication process is illustrated in Figure 11A. Functionalizing MC with PDDA was the first step and then $\mathrm{MoS}_{2}$ nanocubes were decorated onto PDDA-MC by the reduction process of $\left(\mathrm{NH}_{4}\right)_{2} \mathrm{MoS}_{4}$ using the hydrothermal method. L-Cys was demonstrated with amperometric responses plotted in Figure 11B having increased current steps by the elevated concentration of L-Cys (Zheng et al., 2015). He et al. fabricated transparent, flexible RGO thin-film transistors (RGO TFT) to detect fibronectin, which is a type of protein. In Figure 11C, the RGO-based thin-film transistor fabrication is illustrated. GO was first spin-coated on a 3-aminopropyltriethoxysilane (APTES)-modified PET substrate. The APTES helps GO to be adsorbed to the substrate and prevents aggregation. Then, GO was reduced with hydrazine vapor to obtain RGO films. For selectivity, the RGO channel was functionalized with 1-pyrenebutanoic acid succinimidyl ester, which acted as a linker molecule to seize the proteins in the buffer solution. As shown in Figure 11D, the current of the functionalized RGO channel decreased as the fibronectin was added. This is due to the $\mathrm{n}$-doping effect of fibronectin. Also, $\mathrm{He}$ et al. tested the detection of avidin with other functional groups. The RGO channel was coated with polyethyleneimine (PEI) and polyethylene glycol (PEG) and then biotinylated using biotin-Nhydroxysuccinimide ester (He et al., 2011). Mukherjee et al. used a liquid-gated FET graphene decorating aptamer with the linker of the pyrene group to detect adenosine triphosphate (ATP) (Mukherjee et al., 2015). Ohno et al. also designed aptamermodified graphene to detect immunoglobulin $\mathrm{E}$ (IgE), and other proteins such as bovine serum albumin (BSA) and streptavidin (SA) had no response to the device (Ohno et al., 2010).

\section{CONCLUSION AND PERSPECTIVE}

The promising physical and chemical properties of 2D materials have encouraged lots of studies on developing highly selective ion/molecule sensors utilizing surface defects, large specific surface area, and easy surface functionalization. Many researchers are seeking to use $2 \mathrm{D}$ materials as ion/molecule sensors for various reasons such as environmental monitoring, real-life healthcare, and food intake applications. Although studies on $2 \mathrm{D}$ material-based ion/molecule sensors are in 
the early stage compared to oxide-based sensors (Figure 12), various approaches to achieve high selectivity using $2 \mathrm{D}$ materials summarized in this review will benefit when appropriate sensor platform for IoT applications is developed and will result in the realization of e-tongue or taste sensors. To fulfill the advent of taste sensors or e-tongue, the exact one-to-one correspondence of the sensor and target material should be available and the device should discriminate the composition of substance precisely. Despite these efforts, they are still far behind commercialization and will still face lots of obstacles toward usage in the real world. The highly selective receptors with high reliability should be discovered, and reproducible sensor fabrication with a uniform sensor performance should be secured for mass production. In particular, targeting substances in liquid media causes difficulties in developing suitable sensor structures for mobile application and further studies are necessary.

\section{REFERENCES}

Ali, I. (2012). New generation adsorbents for water treatment. Chem. Rev. 112, 5073-5091. doi: 10.1021/cr300133d

Aliprandi, A., Pakulski, D., Ciesielski, A., and Samorì, P. (2017). Punctured twodimensional sheets for harvesting blue energy. ACS Nano 11, 10654-10658. doi: 10.1021/acsnano.7b06657

Allen, B. L., Kichambare, P. D., and Star, A. (2007). Carbon nanotube field-effect-transistor-based biosensors. Adv. Mater. 19, 1439-1451. doi: 10.1002/adma.200602043

Ang, P. K., Chen, W., Wee, A. T. S., and Loh, K. P. (2008). Solution-gated epitaxial graphene as $\mathrm{pH}$ sensor. J. Am. Chem. Soc. 130, 14392-14393. doi: $10.1021 /$ ja805090z

Bai, H., Li, C., Wang, X., and Shi, G. (2010). A pH-sensitive graphene oxide composite hydrogel. Chem. Commun. 46, 2376-2378. doi: 10.1039/c000051e

Berger, C., Song, Z., Li, X., Wu, X., Brown, N., Naud, C., et al. (2006). Electronic confinement and coherence in patterned epitaxial graphene. Science 312, 1191-1196. doi: 10.1126/science.1125925

Boukhvalov, D. W., and Katsnelson, M. I. (2008). Modeling of graphite oxide. J. Am. Chem. Soc. 130, 10697-10701. doi: 10.1021/ja8021686

Butler, S., Hollen, S., Cao, L., Cui, Y., Gupta, J., Gutierrez, H., et al. (2013). Progress, challenges, and opportunities in two-dimensional materials beyond graphene. ACS Nano 7, 2898-2926. doi: 10.1021/nn400280c

Cai, S., Han, Q., Qi, C., Lian, Z., Jia, X., Yang, R., et al. (2016). $\mathrm{Pt}_{74} \mathrm{Ag}_{26}$ nanoparticle-decorated ultrathin $\mathrm{MoS}_{2}$ nanosheets as novel peroxidase mimics for highly selective colorimetric detection of $\mathrm{H}_{2} \mathrm{O}_{2}$ and glucose. Nanoscale 8, 3685-3693. doi: 10.1039/C5NR08038J

Cai, W., Piner, R. D., Stadermann, F. J., Park, S., Shaibat, M. A., Ishii, Y., et al. (2008). Synthesis and solid-state NMR structural characterization of 13C-labeled graphite oxide. Science 321, 1815-1817. doi: 10.1126/science.11 62369

Castellanos-Gomez, A., Agrait, N., and Rubio-Bollinger, G. (2010). Optical identification of atomically thin dichalcogenide crystals. Appl. Phys. Lett. 96, 213116. doi: 10.1063/1.3442495

Chen, J.-L., and Yan, X.-P. (2011). Ionic strength and $\mathrm{pH}$ reversible response of visible and near-infrared fluorescence of graphene oxide nanosheets for monitoring the extracellular pH. Chem. Commun. 47, 3135-3137. doi: $10.1039 / \mathrm{c} 0 \mathrm{cc} 03999 \mathrm{c}$

Cheng, Z., Li, Q., Li, Z., Zhou, Q., and Fang, Y. (2010). Suspended graphene sensors with improved signal and reduced noise. Nano Lett. 10, 1864-1868. doi: $10.1021 / \mathrm{nl} 100633 \mathrm{~g}$

Choi, Y. R., Yoon, Y.-G., Choi, K. S., Kang, J. H., Shim, Y.-S., Kim, Y. H., et al. (2015). Role of oxygen functional groups in graphene oxide for reversible room-temperature $\mathrm{NO}_{2}$ sensing. Carbon 91, 178-187. doi: 10.1016/j.carbon.2015.04.082

\section{AUTHOR CONTRIBUTIONS}

The manuscript was written through contributions of all authors. All authors have given approval to the final version of the manuscript.

\section{FUNDING}

This work was financially supported by the Basic Science Research Program (2017R1A2B3009135), Future Material Discovery Program (2016M3D1A1027666), and the Nano-Material Technology Development Program (2016M3A7B4910) through the National Research Foundation of Korea. JS acknowledges the Global Ph.D. Fellowship Program through the National Research Foundation of Korea funded by the Ministry of Education (2015H1A2A1033701).

Clark, L. C. Jr., and Lyons, C. (1962). Electrode systems for continuous monitoring in cardiovascular surgery. Ann. N. Y. Acad. Sci. 102, 29-45. doi: 10.1111/j.1749-6632.1962.tb13623.x

Cui, Y., Wei, Q., Park, H., and Lieber, C. M. (2001). Nanowire nanosensors for highly sensitive and selective detection of biological and chemical species. Science 293, 1289-1292. doi: 10.1126/science. 1062711

Curreli, M., Zhang, R., Ishikawa, F. N., Chang, H.-K., Cote, R. J., Zhou, C., et al. (2008). Real-time, label-free detection of biological entities using nanowire-based FETs. IEEE Trans. Nanotechnol. 7, 651-667. doi: 10.1109/TNANO.2008.2006165

Eda, G., Lin, Y. Y., Mattevi, C., Yamaguchi, H., Chen, H. A., Chen, I. S., et al. (2010). Blue photoluminescence from chemically derived graphene oxide. Adv. Mater. 22, 505-509. doi: 10.1002/adma.200901996

Fan, F.-R. F., Park, S., Zhu, Y., Ruoff, R. S., and Bard, A. J. (2008). Electrogenerated chemiluminescence of partially oxidized highly oriented pyrolytic graphite surfaces and of graphene oxide nanoparticles. J. Am. Chem. Soc. 131, 937-939. doi: $10.1021 /$ ja8086246

Georgakilas, V., Otyepka, M., Bourlinos, A. B., Chandra, V., Kim, N., Kemp, K. C., et al. (2012). Functionalization of graphene: covalent and noncovalent approaches, derivatives and applications. Chem. Rev. 112, 6156-6214. doi: $10.1021 / \mathrm{cr} 3000412$

Gómez-Navarro, C., Weitz, R. T., Bittner, A. M., Scolari, M., Mews, A., Burghard, M., et al. (2007). Electronic transport properties of individual chemically reduced graphene oxide sheets. Nano Lett. 7, 3499-3503. doi: $10.1021 /$ nl072090c

Gorbachev, R. V., Riaz, I., Nair, R. R., Jalil, R., Britnell, L., Belle, B. D., et al. (2011). Hunting for monolayer boron nitride: optical and Raman signatures. Small 7, 465-468. doi: 10.1002/smll.201001628

He, Q., Sudibya, H. G., Yin, Z., Wu, S., Li, H., Boey, F., et al. (2010). Centimeterlong and large-scale micropatterns of reduced graphene oxide films: fabrication and sensing applications. Acs Nano 4, 3201-3208. doi: 10.1021/nn10 $0780 \mathrm{v}$

He, Q., Wu, S., Gao, S., Cao, X., Yin, Z., Li, H., et al. (2011). Transparent, flexible, all-reduced graphene oxide thin film transistors. ACS Nano 5, 5038-5044. doi: $10.1021 / \mathrm{nn} 201118 \mathrm{c}$

Homola, J. (2008). Surface plasmon resonance sensors for detection of chemical and biological species. Chem. Rev. 108, 462-493. doi: 10.1021/cr068107d

Huang, J., He, Y., Jin, J., Li, Y., Dong, Z., and Li, R. (2014). A novel glucose sensor based on MoS2 nanosheet functionalized with Ni nanoparticles. Electrochim. Acta 136, 41-46. doi: 10.1016/j.electacta.2014.05.070

Huang, T., Nallathamby, P. D., and Xu, X.-H. N. (2008). Photostable singlemolecule nanoparticle optical biosensors for real-time sensing of single cytokine molecules and their binding reactions. J. Am. Chem. Soc. 130, 17095-17105. doi: 10.1021/ja8068853 
Hummers, W. S. Jr., and Offeman, R. E. (1958). Preparation of graphitic oxide. J. Am. Chem. Soc. 80, 1339-1339. doi: 10.1021/ja01539a017

Itti, L. (2015). New eye-tracking techniques may revolutionize mental health screening. Neuron 88, 442-444. doi: 10.1016/j.neuron.2015.10.033

Jeon, J. M., Kim, T. L., Shim, Y. S., Choi, Y. R., Kwon, K. C., Hong, S. H., et al. (2017). Microscopic evidence for strong interaction between Pd and graphene oxide that results in metal-decoration-induced reduction of graphene oxide. Adv. Mater. 29:1605929. doi: 10.1002/adma.201770100

Jiang, S., Cheng, R., Ng, R., Huang, Y., and Duan, X. (2015). Highly sensitive detection of mercury (II) ions with few-layer molybdenum disulfide. Nano Res. 8, 257-262. doi: 10.1007/s12274-014-0658-x

Jung, I., Dikin, D. A., Piner, R. D., and Ruoff, R. S. (2008). Tunable electrical conductivity of individual graphene oxide sheets reduced at "low" temperatures. Nano Lett. 8, 4283-4287. doi: 10.1021/nl8019938

Kang, X., Wang, J., Wu, H., Aksay, I. A., Liu, J., and Lin, Y. (2009). Glucose oxidase-graphene-chitosan modified electrode for direct electrochemistry and glucose sensing. Biosens. Bioelectr. 25, 901-905. doi: 10.1016/j.bios.2009. 09.004

Kwak, Y. H., Choi, D. S., Kim, Y. N., Kim, H., Yoon, D. H., Ahn, S.-S., et al. (2012). Flexible glucose sensor using CVD-grown graphene-based field effect transistor. Biosens. Bioelectr. 37, 82-87. doi: 10.1016/j.bios.2012.04.042

Kwon, S. S., Yi, J., Lee, W. W., Shin, J. H., Kim, S. H., Cho, S. H., et al. (2015). Reversible and irreversible responses of defect-engineered graphenebased electrolyte-gated $\mathrm{pH}$ sensors. ACS Appl. Mater. Interfaces 8, 834-839. doi: $10.1021 /$ acsami.5b10183

Lei, N., Li, P., Xue, W., and Xu, J. (2011). Simple graphene chemiresistors as pH sensors: fabrication and characterization. Measure. Sci. Technol. 22:107002. doi: 10.1088/0957-0233/22/10/107002

Li, J., Yang, Z., Tang, Y., Zhang, Y., and Hu, X. (2013). Carbon nanotubesnanoflake-like $\mathrm{SnS}_{2}$ nanocomposite for direct electrochemistry of glucose oxidase and glucose sensing. Biosens. Bioelecton. 41, 698-703. doi: 10.1016/j.bios.2012.09.059

Li, P., Zhang, D., Jiang, C., Zong, X., and Cao, Y. (2017). Ultra-sensitive suspended atomically thin-layered black phosphorus mercury sensors. Biosens. Bioelectr. 98, 68-75. doi: 10.1016/j.bios.2017.06.027

Liao, W., Wei, W., Tong, Y., Chim, W. K., and Zhu, C. (2018). Lowfrequency noise in layered $\mathrm{ReS}_{2}$ field effect transistors on $\mathrm{HFO}_{2}$ and its application for $\mathrm{pH}$ sensing. ACS Appl. Mater. Interfaces 10, 7248-7255. doi: $10.1021 /$ acsami.8b00193

Lima, P. R., Santos, W. J., de, C. S., Luz, R., Damos, F. S., and Oliveira, A. B., Goulart, M.O., et al. (2008). An amperometric sensor based on electrochemically triggered reaction: redox-active $\mathrm{Ar}-\mathrm{NO} / \mathrm{Ar}-\mathrm{NHOH}$ from 4-nitrophthalonitrile-modified electrode for the low voltage cysteine detection. J. Electroanalyt. Chem. 612, 87-96. doi: 10.1016/j.jelechem.2007. 09.013

Lin, X., Ni, Y., and Kokot, S. (2016). Electrochemical and bio-sensing platform based on a novel 3D $\mathrm{Cu}$ nano-flowers/layered $\mathrm{MoS}_{2}$ composite. Biosens. Bioelectr. 79, 685-692. doi: 10.1016/j.bios.2015.12.072

Loan, P. T. K., Zhang, W., Lin, C. T., Wei, K. H., Li, L. J., and Chen, C. H. (2014). Graphene/MoS 2 heterostructures for ultrasensitive detection of DNA hybridisation. Adv. Mater. 26, 4838-4844. doi: 10.1002/adma.2014 01084

Luo, J., Jiang, S., Zhang, H., Jiang, J., and Liu, X. (2012). A novel non-enzymatic glucose sensor based on $\mathrm{Cu}$ nanoparticle modified graphene sheets electrode. Analyt. Chim. Acta 709, 47-53. doi: 10.1016/j.aca.2011.10.025

Moses, P. G., Mortensen, J. J., Lundqvist, B. I., and Norskov, J. K. (2009). Density functional study of the adsorption and van der Waals binding of aromatic and conjugated compounds on the basal plane of $\mathrm{MoS}_{2}$. J. Chem. Phys. 130:104709. doi: $10.1063 / 1.3086040$

Mukherjee, S., Meshik, X., Choi, M., Farid, S., Datta, D., Lan, Y., et al. (2015). A graphene and aptamer based liquid gated FET-like electrochemical biosensor to detect adenosine triphosphate. IEEE Trans. Nanobiosci. 14, 967-972. doi: 10.1109/TNB.2015.2501364

Novoselov, S., Jiang, D., Schedin, F., Booth, T. J., Khotkevich, V. V., Morozov, S. V., et al. (2005). Two-dimensional atomic crystals. Proc. Natl. Acad. Sci. U.S.A. 102, 10451-10453. doi: 10.1073/pnas.0502848102

Nowogrodzki, A. (2018). Speaking in code: how to program by voice. Nature 559, 141-142. doi: 10.1038/d41586-018-05588-x
Ohno, Y., Maehashi, K., and Matsumoto, K. (2010). Label-free biosensors based on aptamer-modified graphene field-effect transistors. J. Am. Chem. Soc. 132, 18012-18013. doi: $10.1021 / \mathrm{ja} 108127 \mathrm{r}$

Ohno, Y., Maehashi, K., Yamashiro, Y., and Matsumoto, K. (2009). Electrolytegated graphene field-effect transistors for detecting $\mathrm{pH}$ and protein adsorption. Nano Lett. 9, 3318-3322. doi: 10.1021/nl901596m

Park, S., and Ruoff, R. S. (2009). Chemical methods for the production of graphenes. Nat. Nanotechnol. 4, 217-224. doi: 10.1038/nnano.2009.58

Parlak, O., Incel, A., Uzun, L., Turner, A., and Tiwari, A. (2017). Structuring $\mathrm{Au}$ nanoparticles on two-dimensional $\mathrm{MoS}_{2}$ nanosheets for electrochemical glucose biosensors. Biosens. Bioelectron. 89, 545-550. doi: 10.1016/j.bios.2016.03.024

Peng, H.-I., Strohsahl, C. M., Leach, K. E., Krauss, T. D., and Miller, B. L. (2009). Label-free DNA detection on nanostructured Ag surfaces. ACS Nano 3, 2265-2273. doi: 10.1021/nn900112e

Robinson, J. T., Perkins, F. K., Snow, E. S., Wei, Z., and Sheehan, P. E. (2008). Reduced graphene oxide molecular sensors. Nano Lett. 8, 3137-3140. doi: $10.1021 / \mathrm{nl} 8013007$

Sarkar, D., Liu, W., Xie, X., Anselmo, A. C., Mitragotri, S., and Banerjee, K. (2014). MoS2 field-effect transistor for next-generation label-free biosensors. ACS Nano 8, 3992-4003. doi: 10.1021/nn5009148

Setford, S. J., White, S. F., and Bolbot, J. A. (2002). Measurement of protein using an electrochemical bi-enzyme sensor. Biosens. Bioelectr. 17, 79-86. doi: 10.1016/S0956-5663(01)00264-0

Shadman, A., Rahman, E., and Khosru, Q. D. (2016). Monolayer $\mathrm{MoS}_{2}$ and WSe double gate field effect transistor as super nernst $\mathrm{pH}$ sensor and nanobiosensor. Sens. Bio Sens. Res. 11, 45-51. doi: 10.1016/j.sbsr.2016.08.005

Shahrokhian, S. (2001). Lead phthalocyanine as a selective carrier for preparation of a cysteine-selective electrode. Analyt. Chem. 73, 5972-5978. doi: $10.1021 / \mathrm{ac} 010541 \mathrm{~m}$

Shan, C., Yang, H., Han, D., Zhang, Q., Ivaska, A., and Niu, L. (2010). Graphene/AuNPs/chitosan nanocomposites film for glucose biosensing. Biosens. Bioelectr. 25, 1070-1074. doi: 10.1016/j.bios.2009.09.024

Sheng, Z.-H., Zheng, X.-Q., Xu, J.-Y., Bao, W.-J., Wang, F.-B., and Xia, X.-H. (2012). Electrochemical sensor based on nitrogen doped graphene: simultaneous determination of ascorbic acid, dopamine and uric acid. Biosens. Bioelectr. 34, 125-131. doi: 10.1016/j.bios.2012.01.030

Sholl, D. S., and Lively, R. P. (2016). Seven chemical separations to change the world. Nat. News 532, 435-437. doi: 10.1038/532435a

Sitko, R., Turek, E., Zawisza, B., Malicka, E., Talik, E., Heimann, J., et al. (2013). Adsorption of divalent metal ions from aqueous solutions using graphene oxide. Dalton Trans. 42, 5682-5689. doi: 10.1039/c3dt33097d

Sofue, Y., Ohno, Y., Maehashi, K., Inoue, K., and Matsumoto, K. (2011). Highly sensitive electrical detection of sodium ions based on graphene field-effect transistors. Jpn. J. Appl. Phys. 50:06GE07. doi: 10.1143/JJAP.50.06GE07

Sohn, I.-Y., Kim, D.-J., Jung, J.-H., Yoon, O. J., Thanh, T. N., Quang, T. T., et al. (2013). pH sensing characteristics and biosensing application of solution-gated reduced graphene oxide field-effect transistors. Biosens. Bioelectr. 45, 70-76. doi: 10.1016/j.bios.2013.01.051

Song, Y., Qu, K., Zhao, C., Ren, J., and Qu, X. (2010). Graphene oxide: intrinsic peroxidase catalytic activity and its application to glucose detection. Adv. Mater. 22, 2206-2210. doi: 10.1002/adma.200903783

Stankovich, S., Piner, R. D., Chen, X., Wu, N., Nguyen, S. T., and Ruoff, R. S. (2006). Stable aqueous dispersions of graphitic nanoplatelets via the reduction of exfoliated graphite oxide in the presence of poly (sodium 4-styrenesulfonate). J. Mater. Chem. 16, 155-158. doi: 10.1039/B512799H

Staudenmaier, L. (1898). Verfahren zur darstellung der graphitsäure. Berichte der Deutschen Chemischen Gesellschaft 31, 1481-1487. doi: 10.1002/cber.18980310237

Stern, E., Vacic, A., and Reed, M. A. (2008). Semiconducting nanowire field-effect transistor biomolecular sensors. IEEE Trans. Electron Devices 55, 3119-3130. doi: 10.1109/TED.2008.2005168

Stine, R., Robinson, J. T., Sheehan, P. E., and Tamanaha, C. R. (2010). Realtime DNA detection using reduced graphene oxide field effect transistors. $A d v$. Mater. 22, 5297-5300. doi: 10.1002/adma.201002121

Su, S., Sun, H., Xu, F., Yuwen, L., Fan, C., and Wang, L. (2014). Direct electrochemistry of glucose oxidase and a biosensor for glucose based on a glass carbon electrode modified with $\mathrm{MoS}_{2}$ nanosheets 
decorated with gold nanoparticles. Microchim. Acta 181, 1497-1503. doi: 10.1007/s00604-014-1178-9

Su, S., Zhang, C., Yuwen, L., Liu, X., Wang, L., Fan, C., et al. (2016). Uniform Au@Pt core-shell nanodendrites supported on molybdenum disulfide nanosheets for the methanol oxidation reaction. Nanoscale 8, 602-608. doi: 10.1039/C5NR06077J

Sudibya, H. G., He, Q., Zhang, H., and Chen, P. (2011). Electrical detection of metal ions using field-effect transistors based on micropatterned reduced graphene oxide films. ACS Nano 5, 1990-1994. doi: 10.1021/nn103043v

Sun, C.-L., Lee, H.-H., Yang, J.-M., and Wu, C.-C. (2011). The simultaneous electrochemical detection of ascorbic acid, dopamine, and uric acid using graphene/size-selected Pt nanocomposites. Biosens. Bioelectr. 26, 3450-3455. doi: 10.1016/j.bios.2011.01.023

Sun, H., Chao, J., Zuo, X., Su, S., Liu, X., Yuwen, L., et al. (2014). Gold nanoparticledecorated $\mathrm{MoS}_{2}$ nanosheets for simultaneous detection of ascorbic acid, dopamine and uric acid. RSC Adv. 4, 27625-27629. doi: 10.1039/C4RA04046E

Sun, Z., Zhao, Q., Zhang, G., Li, Y., Zhang, G., Zhang, F., et al. (2015). Exfoliated MoS 2 supported Au-Pd bimetallic nanoparticles with core-shell structures and superior peroxidase-like activities. RSC Adv. 5, 10352-10357. doi: 10.1039/C4RA13575J

Updike, S., and Hicks, G. (1967). The enzyme electrode. Nature 214, 986-988. doi: $10.1038 / 214986 a 0$

Wang, Q., Kalantar-Zadeh, K., Kis, A., Coleman, J., and Strano, M. (2012), Electronics and optoelectronics of two-dimensional transition metal dichalcogenides. Nat. Nanotechnol. 7, 699-712. doi: 10.1038/nnano.2012.193

Wang, X., Zhi, L., and Müllen, K. (2008). Transparent, conductive graphene electrodes for dye-sensitized solar cells. Nano Lett. 8, 323-327. doi: $10.1021 / \mathrm{nl} 072838 \mathrm{r}$

Wang, Y., Shao, Y., Matson, D. W., Li, J., and Lin, Y. (2010). Nitrogen-doped graphene and its application in electrochemical biosensing. ACS Nano 4, 1790-1798. doi: 10.1021/nn100315s

Wang, Z., Zhou, X., Zhang, J., Boey, F., and Zhang, H. (2009). Direct electrochemical reduction of single-layer graphene oxide and subsequent functionalization with glucose oxidase. J. Phys. Chem. C 113, 14071-14075. doi: $10.1021 /$ jp906348x

Wen, Y., Li, F. Y., Dong, X., Zhang, J., Xiong, Q., and Chen, P. (2013). The electrical detection of lead ions using gold-nanoparticle-and DNAzymefunctionalized graphene device. Adv. Healthc. Mater. 2, 271-274. doi: 10.1002/adhm.201200220

Williams, G., Seger, B., and Kamat, P. V. (2008). $\mathrm{TiO}_{2}$-graphene nanocomposites. UV-assisted photocatalytic reduction of graphene oxide. ACS Nano 2, 1487-1491. doi: 10.1021/nn800251f

Wu, H., Wang, J., Kang, X., Wang, C., Wang, D., Liu, J., et al. (2009). Glucose biosensor based on immobilization of glucose oxidase in platinum nanoparticles/graphene/chitosan nanocomposite film. Talanta 80, 403-406. doi: 10.1016/j.talanta.2009.06.054

Wu, S., Zeng, Z., He, Q., Wang, Z., Wang, S. J., Du, Y., et al. (2012). Electrochemically reduced single-layer $\mathrm{MoS}_{2}$ nanosheets: characterization, properties, and sensing applications. Small 8, 2264-2270. doi: 10.1002/smll.201200044

Yang, L., Liu, D., Huang, J., and You, T. (2014). Simultaneous determination of dopamine, ascorbic acid and uric acid at electrochemically reduced graphene oxide modified electrode. Sens. Actuat. B Chem. 193, 166-172. doi: $10.1016 /$ j.snb.2013.11.104
Yin, Z., He, Q., Huang, X., Zhang, J., Wu, S., Chen, P., et al. (2012). Real-time DNA detection using Pt nanoparticle-decorated reduced graphene oxide field-effect transistors. Nanoscale 4, 293-297. doi: 10.1039/C1NR11149C

Yoo, G., Bong, J.-H., Kim, S., Jose, J., and Pyun, J.-C. (2014). Microarray based on autodisplayed Ro proteins for medical diagnosis of systemic lupus erythematosus (SLE). Biosens. Bioelectr. 57, 213-218. doi: 10.1016/j.bios.2014.02.018

Zafir Mohamad Nasir, M., Sofer, Z., and Pumera, M. (2015). Effect of electrolyte $\mathrm{pH}$ on the inherent electrochemistry of layered transition-metal dichalcogenides $\left(\mathrm{MoS}_{2}, \mathrm{MoSe}_{2}, \mathrm{WS}_{2}, \mathrm{WSe}_{2}\right)$. ChemElectroChem 2, 1713-1718. doi: 10.1002/celc.201500259

Zeng, Q., Cheng, J.-S., Liu, X.-F., Bai, H.-T., and Jiang, J.-H. (2011). Palladium nanoparticle/chitosan-grafted graphene nanocomposites for construction of a glucose biosensor. Biosens. Bioelectr. 26, 3456-3463. doi: 10.1016/j.bios.2011.01.024

Zhang, L.-X., Cao, Y.-R., Xiao, H., Liu, X.-P., Liu, S.-R., Meng, Q.-H., et al. (2016). Leverage principle of retardation signal in titration of double protein via chip moving reaction boundary electrophoresis. Biosens. Bioelectr. 77, 284-291. doi: 10.1016/j.bios.2015.09.001

Zhao, G., Ren, X., Gao, X., Tan, X., Li, J., Chen, C., et al. (2011). Removal of $\mathrm{Pb}$ (II) ions from aqueous solutions on few-layered graphene oxide nanosheets. Dalton Trans. 40, 10945-10952. doi: 10.1039/c1dt1 1005 e

Zheng, Z., Feng, Q., Li, J., and Wang, C. (2015). The p-type $\mathrm{MoS}_{2}$ nanocube modified poly (diallyl dimethyl ammonium chloride)-mesoporous carbon composites as a catalytic amplification platform for electrochemical detection of L-cysteine. Sens. Actuat. B Chem. 221, 1162-1169. doi: 10.1016/j.snb.2015.07.069

Zhong, X., Yang, H., Guo, S., Li, S., Gou, G., Niu, Z., et al. (2012). In situ growth of $\mathrm{Ni}-\mathrm{Fe}$ alloy on graphene-like $\mathrm{MoS}_{2}$ for catalysis of hydrazine oxidation. J. Mater. Chem. 22, 13925-13927. doi: 10.1039/c2jm3 $2427 \mathrm{j}$

Zhou, G., Chang, J., Pu, H., Shi, K., Mao, S., Sui, X., et al. (2016). Ultrasensitive mercury ion detection using DNA-functionalized molybdenum disulfide nanosheet/gold nanoparticle hybrid fieldeffect transistor device. ACS Sens. 1, 295-302. doi: 10.1021/acssensors. 5 b00241

Zhu, Y., Murali, S., Cai, W., Li, X., Suk, J. W., Potts, J. R., et al. (2010). Graphene and graphene oxide: synthesis, properties, and applications. Adv. Mater. 22, 3906-3924. doi: 10.1002/adma.201001068

Zhu, Y., Wang, C., Petrone, N., Yu, J., Nuckolls, C., Hone, J., et al. (2015). A solid dielectric gated graphene nanosensor in electrolyte solutions. Appl. Phys. Lett. 106:123503. doi: $10.1063 / 1.4916341$

Conflict of Interest: The authors declare that the research was conducted in the absence of any commercial or financial relationships that could be construed as a potential conflict of interest.

Copyright (c) 2019 Lee, Suh and Jang. This is an open-access article distributed under the terms of the Creative Commons Attribution License (CC BY). The use, distribution or reproduction in other forums is permitted, provided the original author(s) and the copyright owner(s) are credited and that the original publication in this journal is cited, in accordance with accepted academic practice. No use, distribution or reproduction is permitted which does not comply with these terms. 